University of South Florida

DIGITAL COMMONS

Digital Commons @ University of

@ UNIVERSITY OF SOUTH FLORIDA

South Florida

January 2013

\title{
Effects of Solvent Composition and Hydrogen Pressure on the Catalytic Conversion of 1,2,4,5-Tetrachlorobenzene to Cyclohexane
}

\author{
Margaret Elizabeth Cone \\ University of South Florida, margaret-cone@utulsa.edu
}

Follow this and additional works at: https://digitalcommons.usf.edu/etd

Part of the Environmental Engineering Commons

\section{Scholar Commons Citation \\ Cone, Margaret Elizabeth, "Effects of Solvent Composition and Hydrogen Pressure on the Catalytic Conversion of 1,2,4,5-Tetrachlorobenzene to Cyclohexane" (2013). USF Tampa Graduate Theses and Dissertations.}

https://digitalcommons.usf.edu/etd/4655

This Thesis is brought to you for free and open access by the USF Graduate Theses and Dissertations at Digital Commons @ University of South Florida. It has been accepted for inclusion in USF Tampa Graduate Theses and Dissertations by an authorized administrator of Digital Commons @ University of South Florida. For more information, please contact digitalcommons@usf.edu. 
Effects of Solvent Composition and Hydrogen Pressure on the Catalytic Conversion of 1,2,4,5-Tetrachlorobenzene to Cyclohexane

by

\author{
Margaret E. Cone
}

\begin{abstract}
A thesis submitted in partial fulfillment
of the requirements for the degree of

Master of Science in Environmental Engineering Department of Civil and Environmental Engineering

College of Engineering

University of South Florida
\end{abstract}

Major Professor: Jeffrey Cunningham, Ph.D.

Qiong Zhang, Ph.D.

John Kuhn, Ph.D.

Date of Approval:

July 9, 2013

Keywords: Hydrodehalogenation, Hydrogenation, Palladium, Rhodium, Remediation

Copyright (C) 2013, Margaret E. Cone 


\section{DEDICATION}

This thesis is dedicated to my parents. My mother's unwavering love and support has made the completion of this thesis possible. While my father will not have the opportunity to read this manuscript, he always encouraged my scholastic endeavors and taught me a love of learning that has followed me throughout my life. His life and scholarship will always be a source of inspiration for me. 


\section{ACKNOWLEDGMENTS}

This thesis would not have been possible without my advisor, Dr. Jeffrey Cunningham, who has provided guidance and mentorship throughout my graduate school experience. I am deeply grateful for his positive attitude, critical eye for detail, and continued support throughout this process. I would also like to thank Dr. James Mihelcic, who inspired me to make the transition to the field of environmental engineering, and who has provided continued support over the past two years. Lastly, I would like to thank the members of my committee for the time they invested in reviewing my work.

This work is supported in part by the National Science Foundation under Grant No. DUE 0965743. Any opinions, findings, conclusions, or recommendations expressed in this thesis are those of the author and do not necessarily reflect the views of the National Science Foundation. 


\section{TABLE OF CONTENTS}

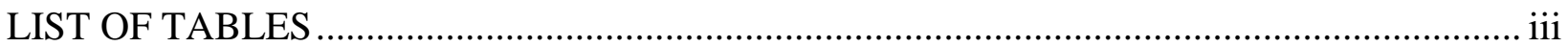

LIST OF FIGURES ……………………………............................................................ iv

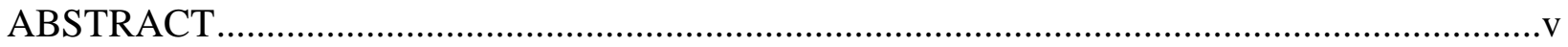

CHAPTER 1: MOTIVATION, INTRODUCTION, AND OBJECTIVES …................................

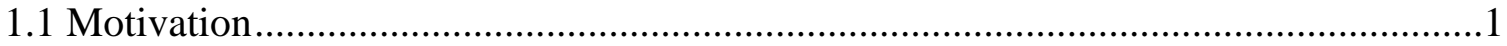

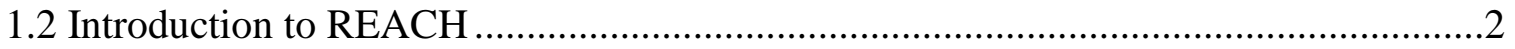

1.3 Objectives .................................................................................................

CHAPTER 2: BACKGROUND ON REACH AND CATALYTIC TREATMENT …...................5

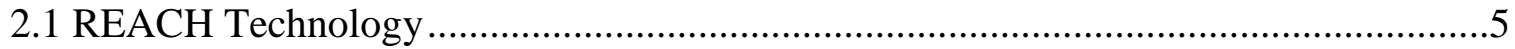

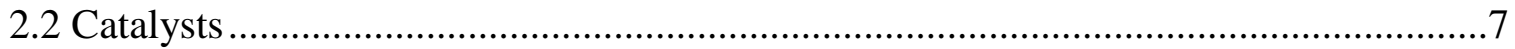

2.3 Pd-Catalyzed Hydrodehalogenation of Hydrophobic Organic Compounds...................9

2.4 Rh-Catalyzed Hydrogenation of Aromatic Compounds .............................................10

2.5 Factors Influencing Heterogeneous Catalytic Reaction Rates.....................................11

2.6 Heterogeneous Catalyst Deactivation ...................................................................13

2.6.1 Introduction to Catalyst Deactivation ...........................................................13

2.6.2 Chemical Deactivation Mechanisms.........................................................14

2.6.3 Catalyst Regeneration and Prevention of Catalyst Deactivation ....................16

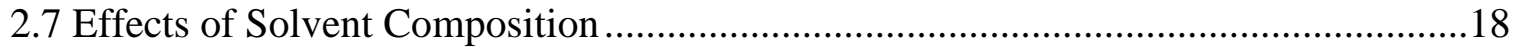

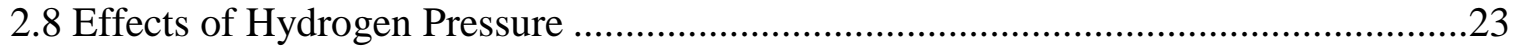

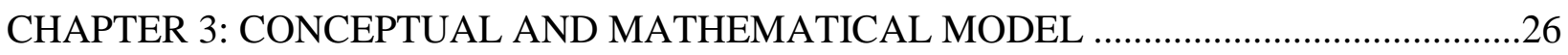

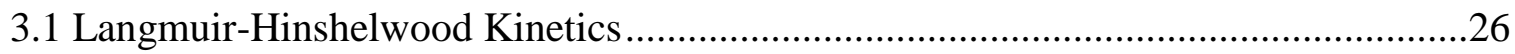

3.2 Hydrodehalogenation Model .............................................................................27

3.3 Hydrogenation Model ..........................................................................................

3.4 Hypothesized Effects of Hydrogen Pressure and Solvent Composition........................32

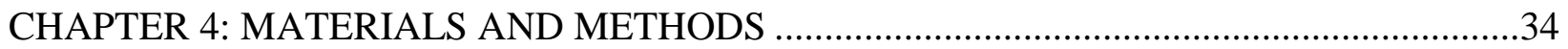

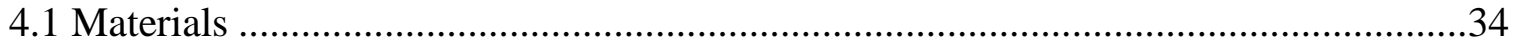

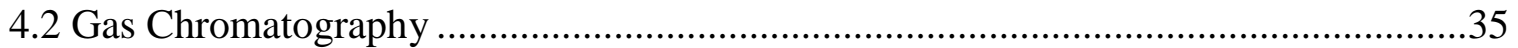

4.3 Preparation and Analysis of TeCB …………….......................................................

4.3.1 Preparation of Stock Solutions and Standards ………...................................36

4.3.2 ECD Analysis of TeCB ..........................................................................

4.3.3 TeCB Calibration Plot Development …………........................................38

4.4 Preparation and Analysis of Cyclohexane and Benzene...............................................39 
4.4.1 Preparation of Stock Solutions and Standards .........................................39

4.4.2 FID Analysis of Cyclohexane and Benzene ............................................40

4.4.3 Cyclohexane and Benzene Calibration Plot Development ..........................41

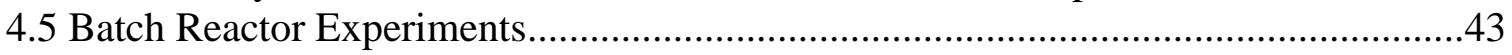

4.5.1 Batch Reactor General Procedure .......................................................43

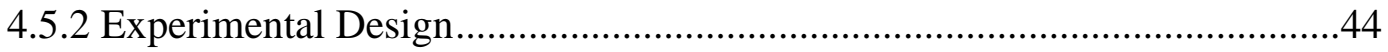

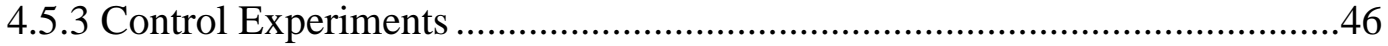

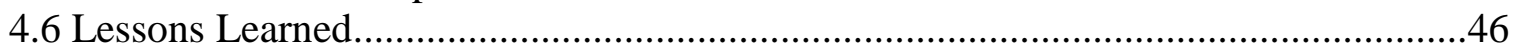

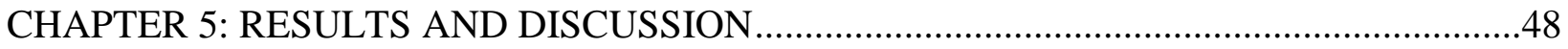

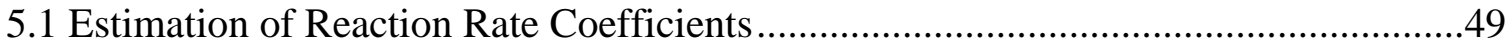

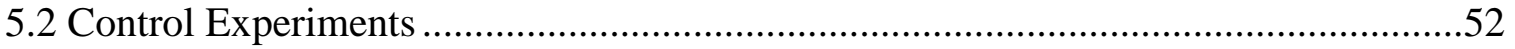

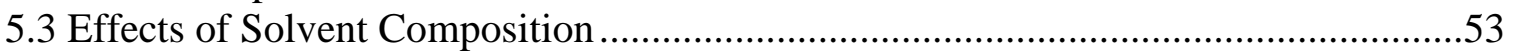

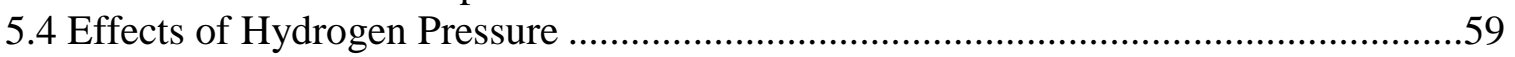

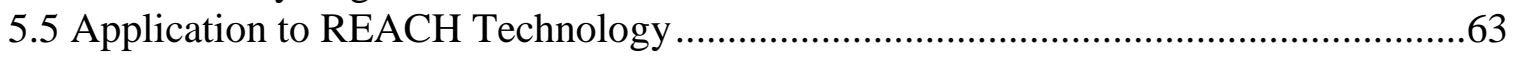

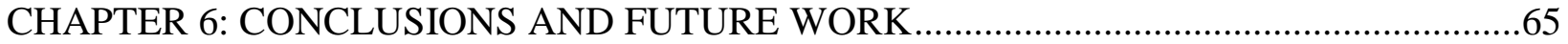

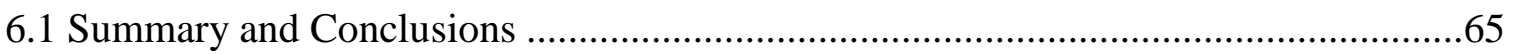

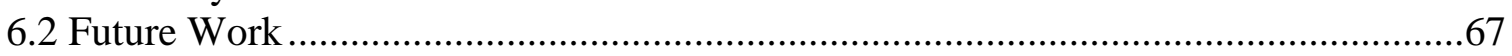

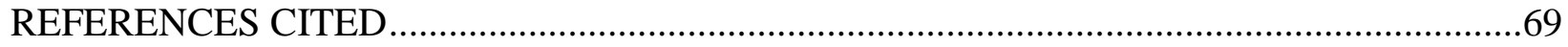

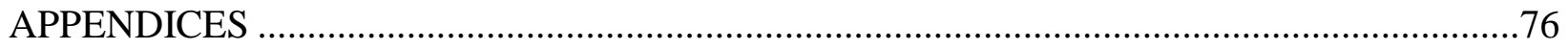

Appendix A: Electron Capture Detector Gas Chromatographic Methods.......................77

Appendix B: Flame Ionization Detector Gas Chromatographic Methods ........................78 


\section{LIST OF TABLES}

Table 4.1. Chemical and Catalyst Specifications and Suppliers...........................................35

Table 4.2. Gas Chromatograph Capillary Column Specifications ............................................36

Table 4.3. Batch Experiment Run Times and Gas Chromatographic Methods..........................45

Table 5.1. Average Initial TeCB Concentrations and Mass Recovery ......................................51

Table 5.2. Estimated Rate Coefficients and Reaction Times for Solvent Composition

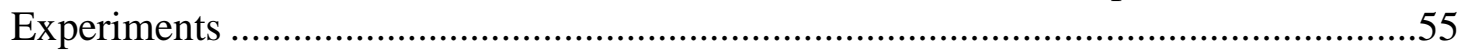

Table 5.3. Estimated Rate Coefficients and Reaction Times for Hydrogen Pressure

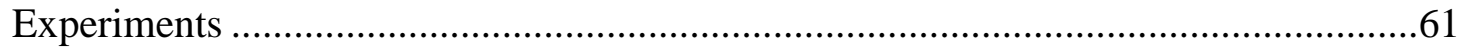

Table A-1. TeCB Gas Chromatography Analysis Method 1 (Before Replacement of ECD Anode and Column) ......................................................................... 77

Table A-2. TeCB Gas Chromatography Analysis Method 2 (After Replacement of ECD Anode and Column) ............................................................................. 77

Table B-1. Cyclohexane and Benzene Gas Chromatography Analysis Method 1 .....................78

Table B-2. Cyclohexane and Benzene Gas Chromatography Analysis Method 2 .....................78 


\section{LIST OF FIGURES}

Figure 4.1. ECD Chromatogram Depicting TCE and TeCB Peaks ...............................................38

Figure 4.2. Calibration Plot for TeCB in 50:50 Water/Ethanol Solvent (Vol:Vol, Before Mixing)

Figure 4.3. FID Chromatogram Depicting Ethanol, Cyclohexane, and Benzene Peaks

Figure 4.4. Calibration Plot for Cyclohexane in 33:67 Water/Ethanol Solvent (Vol:Vol, Before Mixing)

Figure 4.5. Calibration Plot for Benzene in 33:67 Water/Ethanol Solvent (Vol:Vol, Before Mixing)

Figure 5.1. Batch Reactor Experiments for 33:67 Water/Ethanol Solvent Composition and 50 Psi Hydrogen Pressure

Figure 5.2. Batch Reactor Experiments for 50:50 Water/Ethanol Solvent Composition and 50 Psi Hydrogen Pressure

Figure 5.3. Batch Reactor Experiments for 67:33 Water/Ethanol Solvent Composition and 50 Psi Hydrogen Pressure

Figure 5.4. Batch Reactor Experiments for 50:50 Water/Ethanol Solvent Composition and 30 Psi Hydrogen Pressure

Figure 5.5. Batch Reactor Experiments for 50:50 Water/Ethanol Solvent Composition and 10 Psi Hydrogen Pressure 


\begin{abstract}
Halogenated hydrophobic organic compounds (HHOCs) such as 1,2,4,5tetrachlorobenzene (TeCB) present a threat to both human health and the environment. The common occurrence and recalcitrant nature of HHOCs as soil contaminants necessitate an effective soil remediation method. Wee and Cunningham $(2008,2011,2013)$ proposed a cleanup technology called Remedial Extraction and Catalytic Hydrodehalogenation (REACH), which pairs solvent extraction of HHOC contaminants from soil with catalytic hydrodehalogenation to destroy contaminants. Wee and Cunningham $(2008,2011,2013)$ utilized a palladium (Pd) catalyst to hydrodehalogenate TeCB to benzene. However, benzene is still a toxic contaminant. Prior research has demonstrated that Pd-catalyzed hydrodehalogenation $(\mathrm{HDH})$ can be paired with Rh-catalyzed hydrogenation to transform TeCB to cyclohexane, which is a less toxic end product (Osborn 2011; Ticknor 2012). However, there remains a need to quantify the effects of different operating conditions on the catalytic reaction rates upon which the technology relies.

It was hypothesized that (1) an increased ratio of water to ethanol in water/ethanol solvents would increase the reaction rates of both $\mathrm{Pd}$-catalyzed $\mathrm{HDH}$ and $\mathrm{Rh}$-catalyzed hydrogenation, and (2) catalytic reaction rates would be constant above a hydrogen pressure threshold, but would decrease with decreasing hydrogen pressure beneath the threshold. Thus, the objective of this thesis was to contribute to the development of optimal operating parameters for the REACH technology by quantifying the effects of solvent composition and hydrogen
\end{abstract}


pressure on the catalytic conversion of TeCB to cyclohexane in water/ethanol solvents in a batch reactor.

Complete conversion of TeCB to cyclohexane was achieved at all experimental conditions tested. The data were consistent with an apparent first-order kinetics model where Pdcatalyzed HDH and Rh-catalyzed hydrogenation occur in series. The effects of three water/ethanol solvent compositions $(33: 67,50: 50,67: 33)$ were investigated at 50 psi hydrogen pressure. HDH rate coefficients increased monotonically with an increasing fraction of water in the solvent. When the water fraction in the solvent was increased from $50 \%$ to $67 \%$, a larger $\mathrm{HDH}$ rate coefficient increase was observed than when the water fraction was increased from $33 \%$ to $50 \%$. In both cases, the observed increases were statistically significant at a $95 \%$ confidence level. For hydrogenation, rate coefficients at $33 \%$ and $50 \%$ water were approximately equal. The hydrogenation rate coefficient at $67 \%$ water was much greater than the rate coefficients at $50 \%$ and $33 \%$ water, but the increase was not statistically significant at a $95 \%$ confidence level. The observed time for complete conversion of TeCB to cyclohexane decreased with an increasing fraction of water in the solvent, from 12-18 hours with a 33\% water solvent to 8-12 hours with a 50\% water solvent, and to $1-1.5$ hours with a $67 \%$ water solvent.

The effects of three hydrogen pressures (50 psi, $30 \mathrm{psi}, 10 \mathrm{psi}$ ) were investigated with a 50:50 water/ethanol solvent. HDH rate coefficients increased monotonically with decreasing hydrogen pressure, though the trend was not statistically significant at a $95 \%$ confidence level until the pressure was decreased from 30 psi to 10 psi. This trend can be attributed to the displacement of TeCB by hydrogen on the catalyst surface at higher hydrogen pressures. For hydrogenation, the data suggest that rate coefficients are independent of hydrogen pressure in the pressure range of 10-50 psi, since no statistically significant hydrogen pressure effect was 
observed. Complete conversion of $\mathrm{TeCB}$ to cyclohexane was achieved at hydrogen pressures as low as 5 psi.

These findings suggest that a greater fraction of water in the solvent should be utilized in the REACH system when feasible to maximize catalytic reaction rates. These findings also suggest that the REACH system could be operated at hydrogen pressures as low as $5 \mathrm{psi}$, which would further improve the safety of the technology. 


\section{CHAPTER 1: MOTIVATION, INTRODUCTION, AND OBJECTIVES}

\subsection{Motivation}

$1,2,4,5$-Tetrachlorobenzene $(\mathrm{TeCB})$ is one of 31 priority toxic chemicals listed by the U.S. EPA's National Waste Minimization Program (U.S. EPA 2012a). TeCB is a member of a class of chemicals called chlorobenzenes, which are part of a larger group of halogenated hydrophobic organic compounds (HHOCs). HHOCs, which also include chlorinated phenols and polychlorinated biphenyls (PCBs), are among the most common chemicals found at Superfund sites in the United States (U.S. EPA 2011). Due to their hydrophobic and semi-volatile properties, they are highly persistent contaminants typically found in soil that can serve as longterm sources of groundwater contamination (Li and LeBoeuf 2011). Bioaccumulative and toxic, HHOCs present a threat to both human health and the environment. The recalcitrant nature, health implications, and common occurrence of HHOCs as soil contaminants necessitate an effective soil remediation method.

Several methods are available for the remediation of soil contaminated by HHOCs, including incineration, biological reduction, electrokinetic remediation, and solvent extraction paired with activated carbon adsorption. However, significant disadvantages are associated with each technology. Incomplete incineration of HHOCs can generate hazardous air pollutants, such as halogenated dioxins and furans, which must be monitored (Söderström and Marklund 2002; Rivera-Austrui et al. 2011). Hazardous waste incineration is also associated with high operation and maintenance costs (Tang 2004). Biological reduction has been shown to successfully reduce 
concentrations of chlorinated organic compounds (Potrawfke et al. 1998; Chen et al. 2010).

However, the challenges of potentially long lag phases as well as the difficulty of maintaining bacterial mass throughout intermittent operations currently limit large-scale use of bioremediation technology (Chaplin et al. 2012).

Electrokinetic remediation and solvent extraction are phase transfer remediation technologies that aim to separate pollutants from soil. Electrokinetic remediation utilizes a direct current to induce pollutant transport, but can be hindered by sorption of contaminants to soil particles (Gomes et al. 2012). Under traditional solvent extraction, HHOCs tend to concentrate in the solvent, but are not destroyed (U.S. EPA 2001). HHOC-contaminated solvents can be cleansed through activated carbon adsorption, a process commonly used to treat contaminated water and gas streams. Unfortunately, both solvent extraction and activated carbon adsorption processes transfer contaminants from one phase to another without destroying them (Lee et al. 2010). Though the soil may be clean after these processes, the resulting contaminated solvents or activated carbon must be properly stored or further treated to prevent future recontamination or human exposure.

\subsection{Introduction to REACH}

To overcome these challenges, Wee and Cunningham (2008) recently introduced the Remedial Extraction and Catalytic Hydrodehalogenation (REACH) technology. Their goal was to develop a technology that would eliminate the production of a secondary waste stream when treating soil for HHOC contamination, and would therefore be a more environmentally friendly soil remediation alternative. The REACH technology combines solvent extraction of contaminants from soil with catalytic hydrodehalogenation (HDH), a process previously applied to contaminated aqueous solutions where contaminants are transformed with the aid of catalysts 
to less toxic end-products (Kovenklioglu et al. 1992; Xia et al. 2004; Mackenzie et al. 2006; Léger et al. 2007; Ordóñez et al. 2010). Wee and Cunningham (2008) successfully demonstrated that TeCB could be transformed into benzene using palladium-catalyzed HDH in a water-andethanol solvent under mild hydrogen gas pressure. However, though benzene is less toxic than TeCB, it is still one of the EPA's priority pollutants and is not an acceptable end-product for the technology (U.S. EPA 2012b).

Recent work by Ticknor (2012) and Osborn (2011) sought to improve the REACH technology by further transforming benzene to cyclohexane, which is a more benign endproduct. Osborn (2011) succeeded in combining rhodium-catalyzed hydrogenation with palladium-catalyzed $\mathrm{HDH}$ in a single reactor to completely transform TeCB to cyclohexane. Ticknor (2012) demonstrated that TeCB HDH reactions rates could be greatly enhanced through the use of powdered palladium catalyst instead of the pelletized catalyst employed by both Wee and Cunningham (2008, 2011, 2013) and Osborn (2011). However, Ticknor (2012) used a water/ethanol solvent composition of 67:33 instead of the 50:50 solution utilized by Osborn, and solvent composition has been previously suggested to influence HDH reaction rates (Wee and Cunningham 2008). Therefore, it is not clear whether Ticknor (2012) saw increased HDH rates due to the powdered palladium catalyst, the increased ratio of water in the water/ethanol solvent, or both. However, Ticknor (2012) also observed an increase in the hydrogenation reaction rate compared to Osborn (2011). Since palladium catalysts had not been previously shown to contribute to the hydrogenation of benzene to cyclohexane, it is believed that Ticknor (2012) observed a solvent effect.

Wee and Cunningham (2008) previously found that solvent composition significantly affected the rate of Pd-catalyzed HDH of TeCB. Specifically, Wee and Cunningham (2008) 
observed an increase in the $\mathrm{HDH}$ reaction rate with an increase of the proportion of water to ethanol in the solvent. However, Wee and Cunningham (2008) did not explore Rh-catalyzed hydrogenation of benzene. Thus, there remains a need to systematically investigate the effects of solvent composition on both hydrodehalogenation and hydrogenation reactions. Additionally, since hydrogen is a reactant in both the $\mathrm{HDH}$ of $\mathrm{TeCB}$ and the hydrogenation of benzene, hydrogen pressure is another operating condition that has the potential to influence catalytic reaction rates and warrants investigation.

\subsection{Objectives}

The REACH technology has only been assessed at the laboratory scale (Wee and Cunningham 2011). To move towards commercial viability, key factors that must be addressed include catalyst longevity, solvent longevity, and establishment of optimal operating conditions. With respect to operating conditions, it is hypothesized that (1) an increased ratio of water to ethanol in the solvent will increase the reaction rates of both Pd-catalyzed hydrodehalogenation and $\mathrm{Rh}$-catalyzed hydrogenation, and (2) catalytic reaction rates will remain constant above a hydrogen pressure threshold, but will decrease with decreasing hydrogen pressure beneath the threshold. The conceptual and mathematical model that formed the basis for these hypotheses is developed in Chapter 3.

Thus, the objective of this research is to contribute to the determination of optimal operating conditions for the transformation of 1,2,4,5-tetrachlorobenzene into cyclohexane via the REACH process by quantifying the effects of solvent composition and hydrogen pressure on Pd-catalyzed hydrodehalogenation and Rh-catalyzed hydrogenation reaction rates. 


\section{CHAPTER 2: BACKGROUND ON REACH AND CATALYTIC TREATMENT}

The objective of this section is to provide a background on prior research and development of REACH technology as well as related catalytic treatment. The specific catalytic reactions upon which the technology relies - Pd-catalyzed hydrodehalogenation and Rhcatalyzed hydrogenation — will be introduced and current research on these reactions will be summarized. Finally, the primary factors that are suggested in literature to influence catalytic reaction rates will be discussed.

\subsection{REACH Technology}

The Remedial Extraction and Catalytic Hydrodehalogenation (REACH) technology was introduced by Wee and Cunningham $(2008,2011,2013)$ as a novel soil remediation technology that pairs solvent extraction with catalytic hydrodehalogenation (HDH) in a semi-continuous system. The system is semi-continuous because it treats individual batches of soil, but has a continuously flowing closed-loop solvent recycle. Catalytic HDH batch experiments (Wee and Cunningham 2008) and combined solvent extraction and catalytic HDH experiments (Wee and Cunningham 2011, 2013) have demonstrated the feasibility of the technology for remediating certain soils contaminated with either pentachlorophenol (PCP) or 1,2,4,5-tetrachlorobenzene (TeCB).

The bench-scale semi-continuous REACH system utilized by Wee and Cunningham (2011) was comprised of a solvent pump and reservoir, a soil column, a hydrogen gas diffusion module, and a catalyst column. The REACH technology utilizes a water-and-ethanol mixture as 
a solvent because it has previously been proven effective (Khodadoust et al. 1999) and is nonhazardous compared to isopropanol and methanol (Concibido et al. 2006).

In the semi-continuous REACH system utilized by Wee and Cunningham (2008), a water-and-ethanol solvent was pumped through the soil column to extract the contaminants from the soil. The contaminated solvent was then pumped through a hydrogen diffusion module before entering a column of supported palladium (Pd-on- $\mathrm{Al}_{2} \mathrm{O}_{3}$ ) catalyst for $\mathrm{HDH}$. While catalyst deactivation prevented the successful treatment of a Florida soil, 90\% of the contaminants (either PCP or TeCB) in a Texas soil were successfully extracted and hydrodehalogenated after one week of treatment in the semi-continuous system (Wee and Cunningham 2011). The catalyst deactivation observed when treating the Florida soil was attributed to fouling by natural organic matter (Wee and Cunningham 2011).

The REACH technology relies on liquid-phase heterogeneous Pd-catalyzed HDH to remove halogens from HHOCs and reduce their toxicity. For this thesis, I selected 1,2,4,5tetrachlorobenzene $(\mathrm{TeCB})$ as the target contaminant, since it had been used in previous research on the REACH system (Wee and Cunningham 2008, 2011, 2013; Osborn 2011; Ticknor 2012). Hydrodehalogenation of TeCB results in the formation of benzene. In $\mathrm{HDH}$ batch experiments conducted by Wee and Cunningham (2008), only low concentrations of 1,2,4-trichlorobenzene were observed as an intermediate reactant that was rapidly converted to benzene. Thus, for the purposes of this thesis, TeCB is considered to be directly converted to benzene.

Osborn (2011) and Ticknor (2012) further developed the REACH technology by adding Rh-catalyzed hydrogenation, which transforms the product of TeCB HDH (benzene) to a more benign end-product (cyclohexane). The relevant catalytic reactions for the conversion of TeCB, assuming molecular hydrogen is supplied as the reductant, are as follows. 
Pd-catalyzed hydrodehalogenation of TeCB to benzene:

$$
\mathrm{C}_{6} \mathrm{H}_{2} \mathrm{Cl}_{4}+4 \mathrm{H}_{2} \stackrel{\text { Pd catalyst }}{\longrightarrow} \mathrm{C}_{6} \mathrm{H}_{6}+4 \mathrm{H}^{+}+4 \mathrm{Cl}^{-}
$$

Rh-catalyzed hydrogenation of benzene to cyclohexane:

$$
\mathrm{C}_{6} \mathrm{H}_{6}+3 \mathrm{H}_{2} \stackrel{\text { Rh catalyst }}{\longrightarrow} \mathrm{C}_{6} \mathrm{H}_{12}
$$

Net reaction for conversion of $\mathrm{TeCB}$ to cyclohexane:

$$
\mathrm{C}_{6} \mathrm{H}_{2} \mathrm{Cl}_{4}+7 \mathrm{H}_{2} \longrightarrow \mathrm{C}_{6} \mathrm{H}_{12}+4 \mathrm{H}^{+}+4 \mathrm{Cl}^{-}
$$

The catalysts and water/ethanol solvent selected for use in the REACH system allow for operation at ambient temperatures and mild hydrogen pressures. The catalysts utilized in previous research on the REACH process are alumina-supported Pd spheres (Wee and Cunningham 2008, 2011, 2013), alumina-supported pelletized Pd (Osborn 2011), and aluminasupported pelletized Rh (Osborn 2011; Ticknor 2012). Ticknor (2012) utilized a powdered palladium catalyst due to a purchasing error. Osborn (2011) and Ticknor (2012) both emphasized the need for future research to determine optimal operational parameters for the REACH system.

\subsection{Catalysts}

Catalysts increase chemical reaction rates by providing an alternative route for reactions to occur that has a lower required energy input (i.e. lower activation energy). Catalysts are commonly employed to promote the hydrodehalogenation and hydrogenation of organic compounds. Catalysts can be generalized into the following three main categories: biological, homogeneous, and heterogeneous (Smith and Notheisz 1999). Biological catalysts or enzymes increase the rates of biological reactions. Homogeneous catalysts, which are typically organometallic complexes, exist in the same phase as the reactant. In contrast, heterogeneous catalysts are in a different phase than the reactant (e.g. a solid catalyst with an aqueous reactant). Heterogeneous catalysts have the advantage of being easily separated from products, and can be 
in the form of bulk metals, supported metals, or metal oxides.(Smith and Notheisz 1999). Thus, heterogeneous catalysts are ideal for the REACH technology, which aims to reduce secondary waste resulting from remediation efforts.

Important catalyst properties include activity, selectivity, stability, and the ability to be regenerated. Catalyst activity can be defined as how quickly reactions proceed to chemical equilibrium in the presence of the catalyst (Satterfield 1991). One measure of catalyst activity is turnover frequency (TOF), which is the number of molecules converted to a desired product per active site on the catalyst surface per unit of time (Satterfield 1991). Selectivity is the ability of a catalyst to accelerate reactions to form a specific desired product (Satterfield 1991), while stability refers to a catalyst's deactivation behavior (Moulijn et al. 2001). Catalyst deactivation can be defined as reduction in a catalyst's activity or selectivity (Munakata et al. 1998), and is one of the practical barriers to large-scale use of catalytic remediation technologies (Chaplin et al. 2012). Finally, since catalysts are vulnerable to deactivation, the regenerative capabilities of catalysts are crucial to the economic viability of REACH and related technologies that utilize catalytic treatment. No single catalyst is ideal for all hydrodehalogenation and hydrogenation reactions. Thus, catalyst selection is critical to successful catalytic treatment of contaminants. Heterogeneous catalysts typically contain a metal, which can be supported on a variety of materials, such as carbon, silica $\left(\mathrm{SiO}_{2}\right)$, or alumina $\left(\mathrm{Al}_{2} \mathrm{O}_{3}\right)$. Transition metals, especially nickel, platinum, palladium, and rhodium, typically have the highest catalytic activity (Smith and Notheisz 1999). The REACH technology currently employs commercially-available pelletized Pd-on- $\mathrm{Al}_{2} \mathrm{O}_{3}$ and $\mathrm{Rh}$-on- $\mathrm{Al}_{2} \mathrm{O}_{3}$ catalysts for hydrodehalogenation and hydrogenation, respectively. The rationale for selecting $\mathrm{Pd}$ and $\mathrm{Rh}$ catalysts is presented in Sections 2.3 and 2.4. 


\subsection{Pd-Catalyzed Hydrodehalogenation of Hydrophobic Organic Compounds}

Catalytic hydrodehalogenation (or reductive dehalogenation) is the replacement of halogen atoms with hydrogen atoms in the presence of a catalyst (Wee 2007), which is usually a noble metal (Concibido et al. 2007). Catalytic hydrodehalogenation (HDH) requires a hydrogen source that serves as a reductant (Wee 2007). Hydrogen gas (i.e. molecular hydrogen) is a common hydrogen source used in $\mathrm{HDH}$, though hydrogen donors such as inorganic and organic salts, palladised iron nanoparticles, and alcohols have also been utilized (Urbano and Marinas 2001; Kopinke et al. 2004). Hydrodehalogenation is pertinent to remediation efforts because decreased numbers of halogen atoms in an organic waste are typically associated with decreased toxicity of the waste (Murena and Gioia 2009). In that sense, HDH can be considered to be a form of detoxification (Xia et al. 2003). While HDH can be conducted in either the gas or liquid phase, only liquid-phase HDH will be discussed in this thesis, since the objective of this research is to advance the REACH technology.

Palladium (Pd) has emerged as a promising catalyst for reductive transformations of a wide variety of contaminants in water due to its relatively high activity, stability, and selectivity (Chaplin et al. 2012). Palladium is also known to be moderately resistant to catalyst poisons, such as chloride ions, that are released during the HDH of chlorinated organic compounds (Augustine 1965; Urbano and Marinas 2001). Hydrogen chloride formed during catalytic hydrodechlorination can adsorb to the active sites on catalyst's surface, which can lead to catalyst deactivation (Xia et al. 2003).

Overall, palladium is considered a highly efficient metal in the catalysis of hydrodechlorination (Janiak and Okal 2009). Studies have shown the successful liquid-phase hydrodechlorination of chlorinated benzenes on a variety of supported heterogeneous palladium 
catalysts, including Pd-on- $\mathrm{Al}_{2} \mathrm{O}_{3}$ (Scüth and Reinhard 1998; Benítez and Del Angel 2000;

Kopinke et al. 2004; Hashimoto et al. 2005; Mackenzie et al. 2006; Gómez-Quero et al. 2010a, 2010b), Pd-on-SiO 2 (Benítez and Del Angel 2000; Jujjuri and Keane 2010), Pd-on-C (Benítez and Del Angel 2000; Yakovlev et al. 2000; Xia et al. 2004; Janiak and Okal 2009), and other supported palladium catalysts (Lassová et al. 1999; Aramendía et al. 2002). Supported heterogeneous palladium catalysts have also been shown to successfully catalyze the HDH of chlorinated phenols (Hoke et al. 1992; Shindler et al. 2001; Yuan and Keane 2003a, 2003b; Pozan and Boz 2008; Xia et al. 2009; Gómez-Quero et al. 2010a, 2010b), chlorinated ethylenes (Kovenklioglu et al. 1992; Munakata et al. 1998; Lowry and Reinhard 2000; Concibido et al. 2005, 2006, 2007; Mackenzie et al. 2006; Ordóñez et al. 2010), and polychlorinated biphenyls (Forni et al. 1997; Kume et al. 2008; Ishihara et al. 2012).

The REACH technology utilizes a Pd-on- $\mathrm{Al}_{2} \mathrm{O}_{3}$ catalyst for the hydrodehalogenation of HHOCs. According to Wu et al. (2012), alumina is a preferred support for hydrodechlorination because it increases the activity of palladium catalysts due to Lewis acid sites on the support. Lewis acid sites capture chlorine atoms that would otherwise poison the catalysts (Wu et al. 2012).

\subsection{Rh-Catalyzed Hydrogenation of Aromatic Compounds}

Hydrogenation is the addition of hydrogen atoms across covalent chemical pi bonds (Smith and Notheisz 1999), and can be used to detoxify aromatic compounds in the presence of a catalyst at ambient temperatures. Hydrogenation of aromatic compounds such as benzene has also been studied as a method to improve the quality and emissions of diesel fuel (Stanislaus and Cooper 1994; Sidhpuria et al. 2009) and as a useful reaction in organic synthesis (Freifelder 1978). While palladium catalysts are commonly utilized to hydrodehalogenate HHOCs, they 
require acidic conditions, high reaction temperatures, and large catalyst-to-reactant ratios to hydrogenate aromatic compounds such as benzene (Freifelder 1978). Léger et al. (2007) determined experimentally that palladium could only hydrogenate benzene at high hydrogen pressures ( $>40$ bar).

Many transition metals can be utilized for hydrogenation, including rhodium, ruthenium, platinum, palladium, and nickel (Maegawa et al. 2009). However, rhodium exhibits the highest activity for benzene hydrogenation and is considered by some to be the most efficient catalyst for the hydrogenation of the aromatic ring (Smith and Notheisz 1999; Maegawa et al. 2009). Additionally, rhodium has long been known to effectively promote the hydrogenation of aromatic compounds at low temperatures and pressures (Augustine 1965). Therefore, a rhodium catalyst was selected for use in the REACH system.

Prior batch experiments conducted by Osborn (2011) and Ticknor (2012) on catalytic treatment in the REACH process demonstrated that supported heterogeneous rhodium catalysts ( $\mathrm{Rh}$-on- $\mathrm{Al}_{2} \mathrm{O}_{3}$ ) can successfully catalyze the hydrogenation of benzene to cyclohexane at ambient temperatures and moderate hydrogen pressure (50 psi) in mixtures of water and ethanol. However, most recent research on heterogeneous $\mathrm{Rh}$-catalyzed hydrogenation of aromatic compounds has focused on the use of nanoparticles, including both rhodium (Harada et al. 2008; Jacinto et al. 2008) and bimetallic rhodium-nickel nanocatalysts (Duan et al. 2013).

\subsection{Factors Influencing Heterogeneous Catalytic Reaction Rates}

A number of factors are identified in literature as having the potential to influence catalytic hydrodehalogenation and hydrogenation reaction rates. These factors include catalyst characteristics, mass transfer limitations, and catalyst transformations and deactivation. In 
addition, operating conditions such as temperature, pressure, and solvent composition have been shown to influence catalytic reaction rates.

Characteristics of heterogeneous catalysts that can influence catalytic reaction rates include metal particle size and particle dispersion (Smith and Notheisz 1999), catalyst support material (Smith and Notheisz 1999; Benítez and Del Angel 2000), metal-support interactions (Singh and Vannice 2001), catalyst coatings (Navon et al. 2012), and additives during catalyst preparation (Augustine 1965; Urbano and Marinas 2001). In addition, catalyst surface area is often related to heterogeneous catalyst activity, with higher surface area correlating with higher activity (Satterfield 1991). However, commercially-available catalysts have been used in prior research on the REACH system, and thus will be utilized in this thesis. Therefore, the influence of catalyst characteristics on $\mathrm{HDH}$ and hydrogenation reaction rates will not be explored in this thesis. Catalyst transformations during catalysis, many of which cause catalyst deactivation, have also been shown to influence reaction rates. Due to the complexity of the subject, catalyst deactivation will be addressed separately in Section 2.6.

Mass transfer limitations can occur both externally at the liquid-catalyst surface and internally within a heterogeneous catalyst's pores due to intraparticle diffusion (Yuan and Keane 2003b; Mackenzie et al. 2006). Mass transfer limitations are known to influence reaction rates, and tend to shift apparent reaction orders towards one, especially for highly active catalysts (Mackenzie et al. 2006). In their study of the hydrodehalogenation and hydrogenation of chlorinated phenols, Yuan and Keane (2003b) found that mass transfer limitations could be minimized by increasing stirring speed, utilizing a less active catalyst with a smaller particle size, utilizing a higher hydrogen gas flow rate, or starting with a higher initial concentration of contaminant in the solvent. 
Experimental conditions such as temperature, pressure, $\mathrm{pH}$, the rate of agitation of the solution, and the catalyst-to-reactant ratio have also been shown to influence catalytic reaction rates. The exact effects, however, depend on the specific catalysts and reactants involved (Augustine 1965). Since the REACH system was designed to operate at ambient temperatures with a stationary catalyst column, the effects of temperature and agitation rate will not be explored in this thesis. Hydrogen pressure effects have been found to be nonlinear and dependent on the specific substrate and catalyst involved (Augustine 1965). Finally, solvent composition is another factor known to influence hydrogenation and hydrodehalogenation reaction rates and catalyst selectivity, though the effects are complex and are highly dependent on the specific catalysts and substrates involved (Augustine 1965; Urbano and Marinas 2001).

Solvent composition and hydrogen pressure are easily manipulated operating parameters that have previously been suggested to potentially influence catalytic HDH and hydrogenation reaction rates (Wee and Cunningham 2008; Osborn 2011; Ticknor 2012). Thus, solvent composition and hydrogen pressure were selected as the variables to be explored by this thesis. A more detailed review of the literature concerning these operating parameters is presented in Sections 2.7 for solvent composition and Section 2.8 for hydrogen pressure.

\subsection{Heterogeneous Catalyst Deactivation}

\subsubsection{Introduction to Catalyst Deactivation}

As mentioned in Section 2.2, catalyst deactivation, or loss of catalyst activity or selectivity, is one of the major practical barriers to large-scale use of catalytic remediation technologies (Chaplin et al. 2012). Catalyst deactivation can be highly dependent on reaction conditions such as temperature, pressure, solvent composition, and the specific reactants 
involved (Moulijn et al. 2001). Thus, a study of the effects of operating parameters on catalytic reaction rates warrants a discussion of catalyst deactivation.

There are multiple mechanisms of heterogeneous catalyst deactivation that can be generally classified as thermal, mechanical, or chemical (Chinchen 1985). High temperatures can lead to deactivation of catalysts through thermal deactivation mechanisms such as sintering or evaporation of the catalyst material (Moulijn et al. 2001). Sintering is the loss of active sites on the catalyst surface due to crystal growth from migration of metal crystallites or atoms, and usually occurs at high temperatures (Lee 1985). Mechanical deactivation mechanisms include crushing and abrasion, which are often related to high pressure conditions (Moulijn et al. 2001). Since the REACH system is designed to operate at relatively low hydrogen pressures and ambient temperatures, thermal and mechanical deactivation mechanisms will not be discussed further.

\subsubsection{Chemical Deactivation Mechanisms}

Chemical changes to the catalyst can result in chemical deactivation. Chemical deactivation mechanisms include catalyst poisoning, fouling, corrosion of the catalyst support, and metal leaching (Moulijn et al. 2001). Chemical deactivation of catalysts can be either reversible or irreversible, depending on the mechanism of deactivation. Catalyst regeneration is possible if the cause of deactivation is reversible. A few catalyst regeneration techniques are presented in Section 2.6.3.

Poisoning can be reversible or irreversible, depending on the specific mechanism involved (Moulijn et al. 2001). Poisoning can be caused by impurities or by-products of catalyzed reactions, where the deactivation is termed self-poisoning (Hegedus and McCabe 1984). However, poisons most commonly cause deactivation through a process called 
competitive adsorption, where poisons adsorb to the catalyst surface and reduce the number of active sites for the desired reaction to occur (Hegedus and McCabe 1984). After adsorption, poisons can alter catalyst surfaces (poison-induced sintering), weaken reactant-catalyst bonds, or both (Hegedus and McCabe 1984). In addition to decreasing catalyst activity, poisons can also decrease catalyst selectivity by preferentially bonding with specific catalyst surface sites (Hegedus and McCabe 1984). Common palladium catalyst poisons include sulfur-containing compounds, heavy metals (e.g. arsenic, mercury, zinc, lead), phosphorus, halides, ammonia, and acetylene (Bartholomew 2001). Navon et al. (2012) note that hydrogen sulfide in the form of either $\mathrm{H}_{2} \mathrm{~S}$ or $\mathrm{HS}^{-}$is one of the strongest palladium poisons. Interestingly, Bartholomew (2001) also lists benzene as a common catalyst poison due to its ability to bond to multiple active sites on catalyst surfaces.

Catalyst fouling is a form of chemical deactivation caused by the deposition of material on a catalyst surface (Moulijn et al. 2001). It differs from poisoning in that a larger amount of material is usually deposited (Chinchen 1985). Catalyst fouling can be caused by mineral deposition (e.g. coke formation), microbial biomass, dissolved organic matter (DOM), redox active metals, and reduced sulfur species that can complex with palladium and other metal catalysts and block active sites on the catalyst surface (Chaplin et al. 2012).

One common form of fouling is the formation of carbonaceous deposits (coke) on catalyst surfaces. However, Chaplin et al. (2012) note that coke formation is usually not a significant deactivation mechanism during $\mathrm{HDH}$ reactions. Microbial biomass fouling is also generally not observed in short-term laboratory studies. Dissolved organic matter (DOM) can also cause catalyst fouling (Kopinke et al. 2010; Chaplin et al. 2012). Kopinke et al. (2010) note that dissolved organic matter is commonly co-extracted from soil with contaminants during 
solvent extraction. This phenomenon was also suggested by Wee and Cunningham (2011). Since the REACH process couples solvent extraction of contaminants from soil with catalytic destruction of the contaminants, the process is vulnerable to DOM fouling.

Certain catalyst poisons, such as the halide ion chloride and its corresponding acid $\mathrm{HCl}$, can impair catalytic activity through multiple mechanisms. The release of chloride ions during hydrodechlorination and the formation of $\mathrm{HCl}$ are known to be detrimental to catalytic reaction rates due to potential competitive adsorption with reactants, altered surface chemistry, and corrosion or dissolution of the catalyst metal and support (Aramendía et al. 2002). More specifically, chloride can foul catalysts by forming metal chlorides on catalyst surfaces that can block active sites; alternatively, metal chlorides can volatilize, thereby intensifying catalyst sintering (Satterfield 1991). Corrosion of the catalyst support or leaching of the metal can also result from highly acidic solutions caused by $\mathrm{HCl}$ production during hydrodechlorination reactions (Forni et al. 1997; Urbano and Marinas 2001). This effect is less pronounced in fixed bed reactors than in batch reactors, where $\mathrm{HCl}$ tends to concentrate around the catalysts as it is formed (Urbano and Marinas 2001).

\subsubsection{Catalyst Regeneration and Prevention of Catalyst Deactivation}

As mentioned in Section 2.6.2, catalyst deactivation can be irreversible or reversible, depending on the mechanism of deactivation (Chinchen 1985). Thermal deactivation (e.g. sintering) is usually irreversible (Bartholomew 2001). The reversibility of poisoning is dependent on how strongly the poison is adsorbed to the surface and whether or not the surface area of the catalyst has been compromised (Satterfield 1991), such as during poison-induced sintering (Chinchen 1985). It should be noted that not all chemical alterations of catalysts are detrimental. For example, Satterfield (1991) notes that the addition of the common catalyst poison 
dihydrogen sulfide $\left(\mathrm{H}_{2} \mathrm{~S}\right)$ can improve hydrogenation selectivity under certain conditions. It should also be noted that some amount of poisoning is usually tolerated in practice due to the difficulty of completely preventing catalyst poisoning (Chinchen 1985).

Some HDH research groups add a base (e.g. sodium hydroxide) to scavenge free halide ions in solution that could potentially act as catalyst poisons and to mitigate leaching caused by the presence of $\mathrm{HCl}$ (Xia et al. 2003; Yuan and Keane 2003a). However, not all HDH research groups agree on the effectiveness of adding bases such as sodium hydroxide (Xia et al. 2003, 2004; Yuan and Keane 2003a) or sodium carbonate (Ordóñez et al. 2010) to aqueous solutions. Catalyst deactivation has been noted to occur from the deposition of chlorinated salts on catalyst surface after base addition (Xia et al. 2004).

Fouling is a deactivation mechanism that lends itself well to catalyst regeneration (Chinchen 1985). Coke formation is generally reversible via low temperature combustion (Bartholomew et al. 2001), though the temperature must be carefully controlled to avoid sintering (Chinchen 1985). Oxidative catalyst regeneration with dilute sodium hypochlorite has been successfully used to regenerate Pd-on- $\mathrm{Al}_{2} \mathrm{O}_{3}$ catalysts by Lowry and Reinhard (2000) and by Wee and Cunningham (2011), though the initial catalyst deactivation mechanisms were not fully elucidated.

Some research groups have taken a preventative approach to catalyst deactivation by attempting to produce fouling-resistant and poison-resistant catalysts. Potential methods for prevention of fouling include selection of a hydrophobic zeolite support, embedding catalysts in polymers, and the use of bimetallic Pd-Au nanoparticles (Chaplin et al. 2012). Kopinke et al. (2010) investigated the prevention of catalyst DOM fouling by coating a Pd-on- $\mathrm{Al}_{2} \mathrm{O}_{3}$ catalyst with hydrophobic silicone polymers, and observed successful fouling prevention for a period of 
one day. Silicone coatings have also been investigated for their potential to protect catalysts from poisoning. Navon et al. (2012) tested the protection of powdered and pelletized Pd-on- $\mathrm{Al}_{2} \mathrm{O}_{3}$ catalysts against sulfite poisoning with a polydimethylsiloxane (PDMS) polymer coating for the powdered catalysts and pore-filling PMDS for the pelletized catalysts. However, they found that the catalyst treatments were only able to provide initial protection against poisoning, since the PDMS coating was believed to become permeable to poisons over time due to a silicone hydrolysis reaction with $\mathrm{HCl}$ produced during hydrodechlorination (Navon et al. 2012).

\subsection{Effects of Solvent Composition}

The selection of a solvent for liquid-phase catalytic reactions can depend on many factors, including the solubility of the reactants in the solvents, availability, cost, and toxicity (Urbano and Marinas 2001). Solvents in liquid-phase catalytic reactions function not only to dissolve reactants, but can also stabilize intermediate reactants, participate in chemical reactions, and influence catalytic reaction rates. Solvent selection has also been shown to influence both the activity and selectivity of heterogeneous catalysts (Akpa et al. 2012). Potentially important solvent parameters include solvent viscosity, dielectric constant, molar volume, boiling point enthalpy of vaporization, and the solubility of molecular hydrogen in the solvent (Gómez-Quero et al. 2010b).

Most liquid-phase catalytic hydrodehalogenation studies on solvent effects focus on the comparison of different types of solvents (e.g. organic vs. aqueous) rather than the effects of water addition to alcohol solvents (Lassová et al. 1999; Bae et al. 2003; Navon et al. 2012). Bae et al. (2003) compared three aprotic solvents (tetrahydrofuran, acetonitrile, and 1,4-dioxane) and a protic solvent (ethanol). Bae et al. (2003) found ethanol to be more effective due to its ability to donate protons that can scavenge poisonous chloride ions and prevent catalyst poisoning and 
deactivation. Similarly, Yakovlev et al. (2000) contend that alcohols such as methanol, isopropanol, and ethanol can function as hydrogen donors for hydrodechlorination reactions. For their investigation of the hydrodechlorination of chlorobenzene over $\mathrm{Pd}-\mathrm{on}-\mathrm{C}$, they proposed that molecular hydrogen and the ethanol solvent can both act as sources of hydrogen for the hydrodechlorination reaction.

In their study of the dechlorination of chlorophenols, Xia et al. (2009) found that water was superior to three organic solvents (ethanol, hexane, and toluene) in facilitating faster dechlorination rates. They hypothesize that $\mathrm{HCl}$, which forms during the dechlorination process, is more soluble in aqueous solution and thus less apt to cause catalyst deactivation by adsorbing to the catalyst surface when water was used as a solvent. Thus, solvent effects can be related to prevention of catalyst deactivation. The cleansing effect of water as a solvent in Pd-catalyzed hydrodechlorination is also noted by Navon et al. (2012). However, alcohols such as methanol and ethanol are the most common solvents used in liquid-phase hydrodehalogenation of halogenated organic compounds (Urbano and Marinas 2001).

Gómez-Quero et al. (2010b) studied solvent effects in the HDH of 2,4-dichlorophenol over Pd-on- $\mathrm{Al}_{2} \mathrm{O}_{3}$ by conducting experiments in benzene, tetrahydrofuran, $\mathrm{n}$-hexane, $\mathrm{n}$-propanol, cyclohexane, methanol, ethanol, and water solvents. They considered solvent dielectric constant (solvent polarity), molar volume, dynamic viscosity, and boiling point enthalpy of vaporization as potentially important solvent properties, but found that only solvent polarity and molar volume were related to the initial reaction rates. Higher dielectric constants and lower molar volumes were both associated with higher HDH rates, though molar volumes had a much lower contribution to increases in the reaction rates. Gómez-Quero et al. (2010b) attributed the relationship of dielectric constants to catalytic reaction rates to ionic forces that stabilized an 
intermediate reactant. Concibido et al. (2006) similarly found that alcohols with higher dielectric constants were associated with faster HDH rates; in their studies, methanol had the faster HDH rate followed by ethanol and isopropanol. However, their findings may also be related to polarity or solubility effects.

Some researchers have found that polar solvents enhance the adsorption of non-polar reactants to catalyst surfaces and non-polar solvents enhance the adsorption of polar reactants to catalyst surfaces; improved adsorption of reactants to catalyst surfaces is thought to promote faster reaction rates (Singh and Vannice 2001; Concibido et al. 2005). Wee and Cunningham (2008) previously established that the ratio of water to ethanol in the water/ethanol solvent utilized in the REACH process was an important operational parameter for Pd-catalyzed HDH due to solvent polarity and solubility effects. Specifically, Wee and Cunningham (2008) compared 50:50, 33:67, and 67:33 water/ethanol solvent compositions (vol:vol, before mixing) for the $\mathrm{HDH}$ of $\mathrm{TeCB}$, and found that faster reaction rates were achieved with a higher fraction of water in the solvent.

Ticknor (2012) studied the effects of a 67:33 water/ethanol solvent on the catalytic conversion of $\mathrm{TeCB}$ to cyclohexane in the same batch reactor system as Wee and Cunningham (2008) and Osborn (2011). Ticknor (2012) found that both HDH and hydrogenation reactions were faster than those observed by Osborn (2011), who used a 50:50 water/ethanol solvent. Specifically, Ticknor (2012) observed the complete conversion of TeCB to cyclohexane within 45 minutes, which is significantly faster than the 12 hour conversion time observed by Osborn (2011). However, Ticknor (2012) used a powdered palladium catalyst instead of the pelletized Pd-on- $\mathrm{Al}_{2} \mathrm{O}_{3}$ catalyst used by Osborn (2011) or the spherical Pd-on- $\mathrm{Al}_{2} \mathrm{O}_{3}$ catalyst utilized by Wee and Cunningham (2008). As mentioned in Section 2.5, increased catalyst surface area is 
known to influence catalyst activity (Satterfield 1991). While the powdered palladium catalyst may have contributed to the increased HDH reaction rate observed by Ticknor (2012), the increased hydrogenation reaction rate was most likely due to the increased fraction of water in the solvent. Additionally, pelletized catalysts are preferable to powdered catalysts for the semicontinuous REACH system due to ease of separation.

In addition to Wee and Cunningham (2008) and Ticknor (2012), other research groups have investigated the effects of varying alcohol and water ratios in solvents on $\mathrm{HDH}$ reaction kinetics (Hoke et al. 1992; Benítez and Del Angel 1999; Xia et al. 2003; Concibido et al. 2005, 2006, 2007; Gómez-Quero et al. 2010a). Xia et al. (2003) found that dechlorination rates and TOFs for polychlorinated phenoxyphenol over Pd-on-C were higher in water/ethanol mixtures than in ethanol alone. However, they did not observe significant differences in reaction times or TOFs with varying water/ethanol volume ratios.

In contrast, Benítez and Del Angel (1999), Concibido et al. (2006, 2007), Wee and Cunningham (2008), and Gómez-Quero et al. (2010a) report higher hydrodehalogenation rates with increasing water percentage in mixed alcohol and water solvents. Benítez and Del Angel (1999) investigated water, methanol, water/methanol, ethanol, and water/ethanol solvents and found that the fastest conversion and initial reaction rates were obtained with a water solvent. They also found that the HDH reaction rate decreased when a 50:50 water-to-ethanol solvent was used. Similarly, Hoke et al. (1992) found that water and 50:50 water/ethanol solvents were superior to pure ethanol in the $\mathrm{HDH}$ of chlorophenols due to the promotion of faster $\mathrm{HDH}$ reaction rates and the extended life of the catalyst.

Concibido et al. $(2005,2006,2007)$ found that the HDH of tetrachloroethylene (PCE) over Pd-on-C in methanol was enhanced by water addition up to a ratio of 50:50 water-to- 
methanol. They also observed improved selectivity of the catalyst with the addition of water. Concibido et al. (2005) determined that water greatly enhanced the absorption of PCE onto the catalyst through decreasing the solubility of PCE in the solvent. They also note that water addition promotes the removal of reaction products adsorbed to the catalyst surface, which is consistent with the contention of Xia et al. (2009) that water has a cleansing effect on catalysts during HDH. Interestingly, Concibido et al. (2005) observed a decreased reaction rate after the water/methanol ratio was increased to $80: 20$ and 100:0, which they attributed to the decreased solubility of $\mathrm{H}_{2}$ gas in the solvent (Concibido et al. 2005). Pozan and Boz (2008) similarly found that the $\mathrm{HDH}$ of 2,3,5-trichlorophenol decreased in a water/methanol solvent when the proportion of water in the solvent was increased above 50\%. However, Pozan and Boz (2008) still found that the $\mathrm{HDH}$ rate was faster in the 50:50 water/methanol solvent compared to pure methanol.

Interestingly, Concibido et al. (2006) obtained different results when adding water to ethanol or isopropanol instead of methanol. They compared HDH rates in alcohol solvents with $0 \%, 20 \%$, and $50 \%$ water addition by volume. While the HDH rate in methanol increased with as low as $20 \%$ water addition, the $\mathrm{HDH}$ rate in ethanol and isopropanol did not increase until at least $50 \%$ water was added. However, they again attribute these findings to the cleansing effect of water that minimizes catalyst deactivation during hydrodechlorination. More specifically, because they add the base $\mathrm{NaOH}$ to the solvent, they contend that water addition increases the solubility of both $\mathrm{NaOH}$ and $\mathrm{NaCl}$, which can form due to the release of chloride ions during hydrodechlorination. Increased $\mathrm{NaCl}$ solubility reduces the likelihood of adsorption of $\mathrm{NaCl}$ to the catalyst surface and subsequent catalyst deactivation. 
While there is limited literature on the effect of solvent composition on Rh-catalyzed hydrogenation of aromatic compounds, some general solvent effects have been identified in the literature for heterogeneously-catalyzed hydrogenation reactions. Some researchers have noted similar solvent effects to those observed for HDH reactions. For example, Fajt et al. (2008) note that hydrogenation solvent effects can result from the differential solubility of hydrogen gas in the solvent and the competitive adsorption of solvent molecules onto catalyst active sites. In addition, Fajt et al. (2008) note that solvent effects can also be caused by chemical interactions between the reactants and the solvent as well as the agglomeration of catalyst particles. However, they did not investigate the use of a mixed water and alcohol solvent.

Akpa et al. (2012) investigated the kinetic effects of varying ratios of isopropanol and water in a solvent for the liquid-phase hydrogenation of 2-butanone over $\mathrm{Ru}$-on- $\mathrm{SiO}_{2}$. They claim that the structure of water-and-alcohol mixtures can control the diffusion of solvated electrons and influence reaction rates, though their results revealed a complex effect. Interestingly, Akpa et al. (2012) observed that the hydrogenation reaction rate did not increase monotonically with the fraction of water in the solvent, but rather increased abruptly at high water molar concentrations ( $>90 \%)$.

\subsection{Effects of Hydrogen Pressure}

The REACH system is designed to operate at low-to-moderate hydrogen pressures $(<50$ psi). Molecular hydrogen is a reactant in both hydrodechlorination and hydrogenation reactions. Therefore, a minimum hydrogen pressure in the reactor is expected to be necessary for complete catalytic conversion of $\mathrm{TeCB}$ to cyclohexane. Additionally, another hydrogen pressure threshold is hypothesized to exist, below which decreased hydrogen pressure in the reactor decreases catalytic reaction rates due to the lower concentration of hydrogen in solution and thus lower 
surface coverage on the catalyst. Above the hypothesized hydrogen pressure threshold, catalytic reaction rates are expected to be independent of hydrogen pressure due to the limited adsorption capacity of the catalyst. This hypothesized hydrogen pressure effect assumes that hydrogen adsorption follows the Langmuir isotherm, as is further explained in Chapter 3.

From the results of their study of the hydrodechlorination of $p$-chlorophenol in water with a palladium catalyst supported by activated carbon cloth, Shindler et al. (2001) argue that hydrogen is a limiting reactant at atmospheric pressure and ambient temperatures due to its lower solubility in water at low hydrogen pressures. This contention suggests that a minimum hydrogen pressure is required for the hydrogen concentration to reach stoichiometric quantities and thus not limit the catalytic reaction rates. However, increased hydrogen pressure has also been shown to decrease reaction selectivity (Augustine 1965).

No studies known to the author focus solely on the effects of hydrogen pressure on the kinetics of catalytic HDH of liquid-phase contaminants. However, some studies have observed the effects of hydrogen pressure on $\mathrm{HDH}$ catalytic reaction rates, and their findings have been highly variable. Kovenklioglu et al. (1992) found the hydrodechlorination of 1,1,2trichloroethane to be independent of hydrogen pressure. Similarly, Wee and Cunningham (2008) found the $\mathrm{HDH}$ of TeCB to be independent of hydrogen pressure above $0.17 \mathrm{MPa}$ (25 psi). Benítez and Del Angel (1999) varied hydrogen pressure from 0-3 kg/cm ${ }^{2}(0-43 \mathrm{psi})$ and similarly found no effect on the HDH of chlorobenzene over Pd-on-C in a 50:50 water/ethanol solvent.

However, Forni et al. (1997) found that increased hydrogen partial pressure decreased catalyst deactivation and thus improved reaction kinetics in their study of the HDH of polychlorinated biphenyls (PCBs) over a Pd-on-C catalyst in a semi-continuous system. Forni et al. (1997) suggest that increased hydrogen pressure increases the competitiveness of hydrogen 
for catalyst sites, which minimizes deactivation caused by the adsorption of chloride ions to the catalyst surface. Yuan and Keane (2003b) also found increased hydrogen pressure to be beneficial, but attributed this effect to the minimization of mass transfer limitations at both the liquid-catalyst interface and within catalyst pores.

Jujjuri and Keane (2010) varied hydrogen partial pressure from 0.046 to 0.925 atm (0.68$13.6 \mathrm{psi}$ ) in their experiments on the $\mathrm{HDH}$ of chlorinated benzenes over $\mathrm{Pd}$-on- $\mathrm{SiO}_{2}$. In contrast to the findings of Benítez and Del Angel (1999), Jujjuri and Keane (2010) determined that excess stoichiometric hydrogen due to increased hydrogen partial pressures actually inhibited the selectivity of $\mathrm{HDH}$ reactions due to competitive adsorption of hydrogen to the catalyst surface and the displacement of intermediate products.

Konuspayev et al. (2009) investigated the temperature and pressure dependence of the kinetics of benzene hydrogenation with Rh-on-C catalysts. They found that benzene hydrogenation kinetics were far more dependent on temperature than hydrogen pressure when pressure was varied from 20-100 bar (290-1450 psi). In this high pressure range, benzene hydrogenation reaction rates increased with increasing hydrogen pressure. However, the hydrogen pressures investigated by Konuspayev et al. (2009) are much higher than those utilized in the REACH system, which was previously operated at 10 psi (Wee and Cunningham 2011). 


\section{CHAPTER 3: CONCEPTUAL AND MATHEMATICAL MODEL}

When introducing the REACH soil remediation technology, Wee and Cunningham (2008) developed a conceptual and mathematical model for the kinetics of Pd-catalyzed hydrodehalogenation (HDH) of 1,2,4,5-tetrachlorobenzene (TeCB). The objectives of this chapter are (1) to introduce the Langmuir-Hinshelwood kinetic model, which provides the framework for the model developed by Wee and Cunningham (2008) and (2) to follow the development of Wee and Cunningham (2008) to obtain kinetic rate expressions for Pd-catalyzed $\mathrm{HDH}$ of TeCB and Rh-catalyzed hydrogenation of benzene in a batch reactor. The model developed in this chapter is applied in Chapter 5 to determine the effects of solvent composition and hydrogen pressure on catalytic $\mathrm{HDH}$ and hydrogenation reaction rate coefficients.

\subsection{Langmuir-Hinshelwood Kinetics}

The TeCB HDH kinetic model developed by Wee and Cunningham (2008) was based on the Langmuir-Hinshelwood model for the kinetics of reactions on a catalyst surface. LangmuirHinshelwood models assume that surface catalytic reactions occur in the following sequence of steps: (1) mass transfer of reactants from the solvent to the catalyst surface, (2) adsorption of reactants to the catalyst surface; (3) reaction on the catalyst surface; (4) desorption of the products of the reaction from the catalyst surface; (5) mass transfer of reaction products to the solvent (Wee and Cunningham 2008). Typically, Langmuir-Hinshelwood models assume there is a single rate-limiting step, which is usually identified as the surface reaction (Satterfield 1991). Adsorption and desorption reactions are expected to be at equilibrium and to follow Langmuir 
adsorption isotherm behavior (Satterfield 1991). In the batch experimental system utilized in this work, mass transfer limitations can be neglected because the reaction vessel is shaken vigorously. Adsorption and desorption reactions can also be assumed to occur more rapidly than the surface reactions (Wee and Cunningham 2008). Thus, the reaction on the catalyst surface can be assumed to be the rate-controlling step for both Pd-catalyzed $\mathrm{HDH}$ and Rh-catalyzed hydrogenation.

The Langmuir adsorption isotherm relates surface coverage of reactants on catalysts to liquid-phase reactant concentrations (Crittenden et al. 2012). The conceptual basis of the Langmuir isotherm is that there are a limited number of adsorption sites available on a catalyst surface, and therefore catalysts have a maximum adsorption capacity (Nazaroff and AlvarezCohen 2001). Based on the Langmuir adsorption isotherm, the sorbed concentration of a chemical species is linearly proportional to the liquid-phase concentration when the liquid-phase concentration is low, and the sorbed concentration of the species is constant when the liquidphase concentration is high (Satterfield 1991).

\subsection{Hydrodehalogenation Model}

Batch reactor experiments, which are described further in Chapter 4, were conducted with a water-and-ethanol solvent and $\mathrm{Pd}$-on- $\mathrm{Al}_{2} \mathrm{O}_{3}$ and $\mathrm{Rh}$-on- $\mathrm{Al}_{2} \mathrm{O}_{3}$ catalysts for TeCB $\mathrm{HDH}$ and benzene hydrogenation, respectively. Wee and Cunningham (2008) developed a TeCB HDH kinetic model for a batch system containing only $\mathrm{Pd}$-on- $\mathrm{Al}_{2} \mathrm{O}_{3}$ catalyst. However, their model development can be followed to obtain kinetic rate expressions for TeCB HDH and benzene hydrogenation in the dual-catalyst batch system utilized for the research presented in this thesis.

The kinetic rate expression for the $\mathrm{HDH}$ of TeCB over a Pd catalyst can be developed as follows. First, let the TeCB HDH rate be defined as the surface reaction rate 


$$
r_{1}=k_{1}^{P d} C_{T}^{P d}\left(C_{H_{2}}^{P d}\right)^{n 1}
$$

where $r_{1}$ is the $\mathrm{HDH}$ reaction rate, $k_{1}^{P d}$ is the $\mathrm{HDH}$ reaction rate coefficient for the surface reaction on the Pd catalyst, $C_{T}^{P d}$ is the concentration of TeCB adsorbed to the Pd catalyst, $C_{H_{2}}^{P d}$ is the concentration of hydrogen adsorbed to the Pd catalyst, and $n 1$ is the reaction order with respect to hydrogen. Based on previous work by Wee and Cunningham (2008) and Osborn (2011), it is assumed that the $\mathrm{HDH}$ reaction is first order with respect to TeCB. A mass balance on TeCB can then be applied to the batch reactor vessel such that

$$
M^{P d} \frac{d C_{T}^{P d}}{d t}+M^{R h} \frac{d C_{T}^{R h}}{d t}+V \frac{d C_{T}}{d t}=-M^{P d} r_{1}
$$

where $M^{P d}$ is the mass of $\mathrm{Pd}$ catalyst, $M^{R h}$ is the mass of Rh catalyst, $C_{T}^{R h}$ is the TeCB concentration on the Rh catalyst, $V$ is the volume of solvent, and $C_{T}$ is the liquid-phase concentration of TeCB. Substituting equation (4) into equation (5) yields

$$
M^{P d} \frac{d C_{T}^{P d}}{d t}+M^{R h} \frac{d C_{T}^{R h}}{d t}+V \frac{d C_{T}}{d t}=-M^{P d} k_{1}^{P d} C_{T}^{P d}\left(C_{H_{2}}^{P d}\right)^{n 1}
$$

Next, it can be assumed that the adsorption of TeCB to Pd and Rh catalyst surfaces is described by the Langmuir adsorption isotherms

$$
\begin{aligned}
C_{T}^{P d} & =q_{T}^{P d} \frac{b_{T}^{P d} C_{T}}{1+b_{T}^{P d} C_{T}} \\
C_{T}^{R h} & =q_{T}^{R h} \frac{b_{T}^{R h} C_{T}}{1+b_{T}^{R h} C_{T}}
\end{aligned}
$$

where $q_{T}^{P d}$ and $q_{T}^{R h}$ are the maximum TeCB adsorption capacities of $\mathrm{Pd}$ and $\mathrm{Rh}$ catalysts, respectively, and $b_{T}^{P d}$ and $b_{T}^{R h}$ are empirical adsorption parameters (Nazaroff and AlvarezCohen, 2001). The liquid-phase concentration of TeCB can be assumed to be in the low 
concentration range of the Langmuir isotherm, where $C_{T}$ is much less than $1 / b_{T}^{P d}$ and $1 / b_{T}^{R h}$. Therefore, at low liquid-phase concentrations, the Langmuir isotherms reduce to

$$
\begin{aligned}
& C_{T}^{P d}=q_{T}^{P d} b_{T}^{P d} C_{T}=K_{T}^{P d} C_{T} \\
& C_{T}^{R h}=q_{T}^{R h} b_{T}^{R h} C_{T}=K_{T}^{R h} C_{T}
\end{aligned}
$$

where $K_{T}^{P d}$ and $K_{T}^{R h}$ are apparent linear partitioning coefficients that describe the equilibrium between the concentration of $\mathrm{TeCB}$ adsorbed to either the Pd catalyst $\left(C_{T}^{P d}\right)$ or the $\mathrm{Rh}$ catalyst $\left(C_{T}^{R h}\right)$ and the liquid-phase concentration of TeCB in the solvent $\left(C_{T}\right)$. Combining equations (6), (9), and (10) yields

$$
\frac{d C_{T}}{d t}=-k_{1}^{P d} \frac{M^{P d} K_{T}^{P d}\left(C_{H_{2}}^{P d}\right)^{n 1}}{M^{P d} K_{T}^{P d}+M^{R h} K_{T}^{R h}+V} C_{T}
$$

The concentration of hydrogen on the Pd catalyst surface $\left(C_{H_{2}}^{P d}\right)$ and on the $\mathrm{Rh}$ catalyst surface $\left(C_{H_{2}}^{P d}\right)$ can also be assumed to be constant over time, since a constant hydrogen pressure was maintained during individual batch reactor experiments and adsorbed reactants are assumed to be in equilibrium with liquid-phase reactants. Therefore, a new $\mathrm{HDH}$ rate coefficient $k_{1}$ can be defined as

$$
k_{1}=k_{1}^{P d} \frac{M^{P d} K_{T}^{P d}\left(C_{H_{2}}^{P d}\right)^{n 1}}{M^{P d} K_{T}^{P d}+M^{R h} K_{T}^{R h}+V}
$$

Equation (11) therefore reduces to

$$
\frac{d C_{T}}{d t}=-k_{1} C_{T}
$$

Therefore, at low liquid-phase concentrations of $\mathrm{TeCB}$, the $\mathrm{HDH}$ reaction should exhibit firstorder kinetics with respect to the liquid-phase concentration of TeCB. The HDH model developed in this section is applied in the interpretation of experimental results in Chapter 5. 


\subsection{Hydrogenation Model}

The model development of Wee and Cunningham (2008), which was applied in Section 3.2 for Pd-catalyzed $\mathrm{HDH}$ of TeCB, can be further applied to the hydrogenation of benzene over a Rh catalyst. Let the benzene hydrogenation rate be defined by the surface reaction rate

$$
r_{2}=k_{2}^{R h} C_{B}^{R h}\left(C_{H_{2}}^{R h}\right)^{n 2}
$$

where $r_{2}$ is the hydrogenation reaction rate, $k_{2}^{R h}$ is the hydrogenation reaction rate coefficient for the Rh-catalyzed surface reaction, $C_{B}^{R h}$ is the concentration of benzene adsorbed to the $\mathrm{Rh}$ catalyst, and $n 2$ is the reaction order with respect to hydrogen. Based on previous work by Osborn (2011), it is assumed that the hydrogenation reaction is first order with respect to benzene. A mass balance on benzene can then be applied to the batch reactor vessel such that

$$
M^{R h} \frac{d C_{B}^{R h}}{d t}+M^{P d} \frac{d C_{B}^{P d}}{d t}+V \frac{d C_{B}}{d t}=M^{P d} r_{1}-M^{R h} r_{2}
$$

where $M^{R h}$ is the mass of Rh catalyst, $M^{P d}$ is the mass of Pd catalyst, $C_{B}^{P d}$ is the concentration of benzene adsorbed to the Pd catalyst, $V$ is the volume of solvent, and $C_{B}$ is the liquid-phase concentration of benzene. Notice that the right-hand side of equation (15) has two terms. The first term represents the formation of benzene by the $\mathrm{HDH}$ of $\mathrm{TeCB}$, while the second term represents the consumption of benzene by hydrogenation. Combining equations (4), (14) and (15) yields

$$
M^{R h} \frac{d C_{B}^{R h}}{d t}+M^{P d} \frac{d C_{B}^{P d}}{d t}+V \frac{d C_{B}}{d t}=M^{P d} k_{1}^{P d} C_{T}^{P d}\left(C_{H_{2}}^{P d}\right)^{n 1}-M^{R h} k_{2}^{R h} C_{B}^{R h}\left(C_{H_{2}}^{R h}\right)^{n 2}
$$

Liquid-phase benzene concentrations are assumed to be on the far left-hand side of the Langmuir isotherm such that the liquid-phase benzene concentration is linearly proportional to the surface concentration of benzene on the Rh catalyst. Therefore, liquid-phase and catalyst surface concentrations of benzene can be related by the expressions 


$$
\begin{aligned}
& C_{B}^{R h}=K_{B}^{R h} C_{B} \\
& C_{B}^{P d}=K_{B}^{P d} C_{B}
\end{aligned}
$$

where $K_{B}^{R h}$ is an apparent linear partitioning coefficient that describes the equilibrium between the liquid-phase concentration of benzene in the water/ethanol solvent $\left(C_{B}\right)$ and the concentration of benzene on the Rh catalyst surface $\left(C_{B}^{R h}\right)$. Similarly, $K_{B}^{P d}$ is an apparent linear partitioning coefficient that describes the equilibrium between $C_{B}$ and $C_{B}^{P d}$. Combining equations (16), (17), and (18) yields

$$
\frac{d C_{B}^{\text {solv }}}{d t}=k_{1}^{P d} \frac{M^{P d} K_{T}^{P d}\left(C_{H_{2}}^{P d}\right)^{n 1}}{M^{R h} K_{B}^{R h}+M^{P d} K_{B}^{P d}+V} C_{T}-k_{2}^{R h} \frac{M^{R h} K_{B}^{R h}\left(C_{H_{2}}^{R h}\right)^{n 2}}{M^{R h} K_{B}^{R h}+M^{P d} K_{B}^{P d}+V} C_{B}
$$

As was previously assumed for the HDH model developed in Section 3.2, the concentrations of hydrogen on the Pd catalyst surface $\left(C_{H_{2}}^{P d}\right)$ and the Rh catalyst surface $\left(C_{H_{2}}^{R h}\right)$ are assumed to be constant during hydrogenation reactions, since pressure is held constant during individual batch reactor experiments and adsorbed reactants are assumed to be in equilibrium with liquid-phase reactants. A new rate coefficient for hydrogenation $\left(k_{2}\right)$ can thus be defined as

$$
k_{2}=k_{2}^{R h} \frac{M^{R h} K_{B}^{R h}\left(C_{H_{2}}^{R h}\right)^{n 2}}{M^{R h} K_{B}^{R h}+M^{P d} K_{B}^{P d}+V}
$$

Combining equations (12), (19), and (20) yields the rate expression

$$
\frac{d C_{B}}{d t}=k_{1} C_{T}-k_{2} C_{B}
$$

A mass balance can similarly be performed on cyclohexane, which is the product of the hydrogenation of benzene, to obtain the rate expression

$$
\frac{d C_{C}}{d t}=k_{2} C_{B}
$$


where $C_{C}$ is the liquid-phase cyclohexane concentration. Equations (13), (21), and (22) together form a kinetic model for the liquid-phase concentrations of TeCB, benzene, and cyclohexane in a batch reactor. The model suggests sequential first-order reaction kinetics, and is applied in the interpretation of batch reactor experiments in Chapter 5.

\subsection{Hypothesized Effects of Hydrogen Pressure and Solvent Composition}

Based on the mathematical models for the kinetics of liquid-phase TeCB HDH and benzene hydrogenation reaction developed in Section 3.2 and Section 3.3, the hypotheses presented in Chapter 1 were developed for the effects of water-to-ethanol solvent composition and hydrogen pressure. Based on the mathematical model developed in this chapter, the ratio of water to ethanol in the water/ethanol solvent was expected to influence reaction rates by influencing the apparent linear partitioning coefficients for TeCB on the Pd catalyst $\left(K_{T}^{P d}\right)$ and benzene on the Rh catalyst $\left(K_{B}^{R h}\right)$. The partitioning coefficients were expected to increase with an increasing fraction of water in the solvent, with a higher percentage of hydrophobic TeCB and benzene adsorbing to the catalyst surface and thus facilitating faster $\mathrm{HDH}$ and hydrogenation reaction rates. From equation (9), it can be seen that as $K_{T}^{P d}$ increases, the apparent rate coefficient for TeCB HDH $\left(k_{1}\right)$ increases. Similarly, it can be seen from equation (17) that as $K_{B}^{R h}$ increases, the apparent rate coefficient for benzene hydrogenation $\left(k_{2}\right)$ correspondingly increases.

Since hydrogen is a reactant in both HDH and hydrogenation, hydrogen pressure effects were hypothesized based on the Langmuir adsorption isotherm. According to the Langmuir isotherm, decreasing hydrogen pressure below a certain hydrogen pressure threshold will decrease the surface coverage of hydrogen on the catalyst. In addition, since the Langmuir adsorption isotherm posits that there are a limited number of sites on the catalyst surface 
available for adsorption, increasing hydrogen pressure above a certain threshold will have no impact on the surface coverage of hydrogen. According to equations (4) and (14), increased surface coverage of reactants (e.g. TeCB, benzene, $\mathrm{H}_{2}$ ) on the catalyst surface will increase catalytic reaction rates. Assuming that increased surface coverage of hydrogen corresponds with increased reaction rates, catalytic $\mathrm{HDH}$ and hydrogenation reaction rates were thus expected to decrease with decreasing hydrogen pressure below a pressure threshold and to be independent of hydrogen pressure above the threshold. 


\section{CHAPTER 4: MATERIALS AND METHODS}

Batch reactor experiments were conducted to investigate the effects of solvent composition and hydrogen pressure on Pd-catalyzed hydrodehalogenation of 1,2,4,5tetrachlorobenzene $(\mathrm{TeCB})$ to benzene and $\mathrm{Rh}$-catalyzed hydrogenation of benzene to cyclohexane. The objective of this chapter is to describe the methods and materials used to conduct the research presented in this thesis.

\subsection{Materials}

All gases were supplied by Airgas (Tampa, FL). An ultra-high purity grade (99.999\%) hydrogen gas cylinder was used as a hydrogen source during batch reactor experiments. A PerkinElmer Clarus 500 gas chromatograph was utilized to quantify concentrations of TeCB, benzene, and cyclohexane in a water/ethanol solvent. The gases used to operate the Clarus 500 gas chromatograph included helium (99.999\%) as a carrier gas, nitrogen (99.999\%) as a make-up gas for the electron capture detector, and hydrogen (99.999\%) and compressed air $\left(20-22 \% \mathrm{O}_{2}\right)$ as fuel for the flame ionization detector.

The catalysts and chemicals used for batch reactor experiments and for gas chromatographic analyses are detailed in Table 4.1. Alumina-supported catalysts were used to maintain consistency with the work of Wee and Cunningham $(2008,2011,2013)$ and Osborn (2011). The supported palladium catalyst used in the hydrodehalogenation of TeCB was 5\% palladium $(\mathrm{Pd})$ by weight on alumina $\left(\mathrm{Al}_{2} \mathrm{O}_{3}\right)$, and will be referred to as $\mathrm{Pd}$-on- $-\mathrm{Al}_{2} \mathrm{O}_{3}$. The supported rhodium catalyst used in the hydrogenation of benzene was $0.5 \%$ rhodium $(\mathrm{Rh})$ by 
weight on alumina, and will be referred to as $\mathrm{Rh}-\mathrm{on}-\mathrm{Al}_{2} \mathrm{O}_{3}$. Catalysts were used as received with no reduction before use. Deionized water was obtained from a Thermo Scientific Barnstead GenPure water purification system.

Table 4.1. Chemical and Catalyst Specifications and Suppliers

\begin{tabular}{|c|c|c|}
\hline Chemical/Catalyst & Specifications & Supplier \\
\hline Ethyl Alcohol (Ethanol) & $\begin{array}{l}\text { 99.5+\%, } 200 \text { proof, } \\
\text { ACS Reagent }\end{array}$ & Acros Organics \\
\hline Pentane & $98 \%$ & Acros Organics \\
\hline Cyclohexane & $99+\%$, ACS Reagent & Sigma-Aldrich \\
\hline Benzene & $\begin{array}{l}>99 \% \text {, thiophene-free, } \\
\text { ACS Reagent }\end{array}$ & Sigma-Aldrich \\
\hline Trichloroethylene (TCE) & $\begin{array}{l}99.5 \% \text { minimum, } \\
\text { ACS Reagent }\end{array}$ & Fisher Scientific \\
\hline 1,2,4,5-Tetrachlorobenzene (TeCB) & $98 \%$ & Sigma-Aldrich \\
\hline Deionized Water & $18.2 \mathrm{M} \Omega * \mathrm{~cm}$ & $\begin{array}{l}\text { Thermo Scientific } \\
\text { Barnstead }\end{array}$ \\
\hline Pd-on- $\mathrm{Al}_{2} \mathrm{O}_{3}$ Catalyst & $\begin{array}{l}5 \mathrm{wt} . \% \text { on alumina, } \\
3 \mathrm{~mm} \text { pellets }\end{array}$ & Alfa Aesar \\
\hline $\mathrm{Rh}$-on- $\mathrm{Al}_{2} \mathrm{O}_{3}$ Catalyst & $\begin{array}{l}0.5 \mathrm{wt} . \% \text { on alumina, } \\
3.2 \mathrm{~mm} \text { pellets }\end{array}$ & Sigma-Aldrich \\
\hline
\end{tabular}

\subsection{Gas Chromatography}

Hydrodehalogenation (HDH) and hydrogenation reactions were monitored during batch reactor experiments, which are described in Section 4.5, by measuring concentrations of TeCB, benzene, and cyclohexane in a water-and-ethanol solvent. Osborn (2011) successfully used gas chromatography (GC) to quantify each chemical on a PerkinElmer Clarus 500 gas chromatograph equipped with an electron capture detector (ECD) and a flame ionization detector (FID). For this project, samples were prepared and analyzed on the same gas chromatograph using similar methods to those developed by Osborn (2011). 
The capillary columns utilized for both FID and ECD analysis are listed in Table 4.2. Column characteristics that can influence chemical elution times are also listed, including column dimensions and the column stationary phase (i.e. the inner lining of the column). Partway through the experimental work, the DB-WAX column, which was connected to the ECD, was damaged during maintenance. The column was replaced with an HP-5 column, which was utilized for the remainder of the experiments. The RTX-1301 column was used for FID analysis for all experiments. The methods used for GC analysis are provided in Appendices A and B.

Table 4.2. Gas Chromatograph Capillary Column Specifications

\begin{tabular}{|c|c|c|c|c|}
\hline Column & Detector & Stationary Phase & $\begin{array}{c}\text { Dimensions } \\
(\text { Length } \times \text { Internal Diameter } \\
\times \text { Film Thickness })\end{array}$ & Supplier \\
\hline RTX-1301 & FID & $\begin{array}{c}6 \% \text { cyanopropyl } \\
\text { phenyl, } 94 \% \text { dimethyl } \\
\text { polysiloxane }\end{array}$ & $30 \mathrm{~m} \times 0.53 \mathrm{~mm} \times 3.0 \mu \mathrm{m}$ & Restex \\
\hline DB-WAX & ECD & $\begin{array}{c}100 \% \text { polyethylene } \\
\text { glycol }\end{array}$ & $30 \mathrm{~m} \times 0.32 \mathrm{~mm} \times 0.25 \mu \mathrm{m}$ & $\begin{array}{c}\mathrm{J} \& \mathrm{~W} \\
\text { Scientific }\end{array}$ \\
\hline HP-5 & ECD & $\begin{array}{c}(5 \% \text {-phenyl)- } \\
\text { methylpolysiloxane. }\end{array}$ & $30 \mathrm{~m} \times 0.32 \mathrm{~mm} \times 0.25 \mu \mathrm{m}$ & $\begin{array}{c}\text { J\&W } \\
\text { Scientific }\end{array}$ \\
\hline
\end{tabular}

\subsection{Preparation and Analysis of TeCB}

\subsubsection{Preparation of Stock Solutions and Standards}

A $2000 \mathrm{mg} / \mathrm{L}$ TeCB stock solution was prepared in a $20 \mathrm{~mL}$ borosilicate glass vial by adding $40 \mathrm{mg}$ of TeCB to $20 \mathrm{~mL}$ of ethanol. Serial dilutions were then performed to prepare standards of $0.1,0.5,1.0,5.0$, and $10 \mathrm{mg} / \mathrm{L}$ TeCB concentrations in mixtures of water and ethanol. The water-to-ethanol solvent concentrations were 33:67, 50:50, or 67:33 (vol:vol, before mixing), depending on the solvent composition being used for experiments at the time. Standards and any remaining stock were stored in a freezer in $20 \mathrm{~mL}$ borosilicate glass vials closed by screw caps with polytetrafluoroethylene (PTFE)-lined septa to minimize volatilization. In 
accordance with the guidelines developed by Osborn (2011), the solutions were stored for a maximum of two weeks.

Injection of water and ethanol into gas chromatographic columns can accelerate column deterioration. It was therefore necessary to extract TeCB from water-and-ethanol solutions into an organic solvent, since the low volatility of TeCB prohibits a headspace injection method. TeCB will preferentially reside in organic solvents due to its relatively high octanol-water partition coefficient and low solubility in water and ethanol (U.S. NLM 2003). Pentane was previously utilized by Osborn (2011) and Ticknor (2012), and thus was selected as the organic solvent for TeCB analysis. A pentane solution spiked with trichloroethylene (TCE) as an internal standard was created through serial dilution. The resulting concentration of TCE in the pentane solution was approximately $1.2 \mathrm{mg} / \mathrm{L}$. The solution was refrigerated in a $250 \mathrm{~mL}$ amber glass bottle affixed with a PTFE-lined screw cap to minimize volatilization.

The TeCB standards were prepared for GC/ECD analysis by placing $2 \mathrm{~mL}$ of each standard in two $5 \mathrm{~mL}$ borosilicate glass vials along with $2 \mathrm{~mL}$ of the TCE-spiked pentane. The resulting ten sample vials were secured with screw caps and shaken on a VWR OS-500 Orbital Shaker at approximately $200 \mathrm{rpm}$ for one hour. The samples were then equilibrated (i.e. allowed to rest undisturbed) for one hour. Finally, $1 \mathrm{~mL}$ of pentane from each sample vial was placed in a $2 \mathrm{~mL}$ PerkinElmer amber glass autosampler vial. The vials were affixed with screw caps with pierceable septa and placed in the gas chromatograph autosampler tray for GC/ECD analysis.

\subsubsection{ECD Analysis of TeCB}

Due to the ECD's sensitivity to electronegative chemical species, which include chlorinated organic compounds, the ECD was selected to analyze TeCB concentrations. As described in Section 4.3.1, TeCB was extracted into a pentane solution spiked with 
trichloroethylene (TCE) as an internal standard. Extracted standards were analyzed in the order of lowest to highest $\mathrm{TeCB}$ concentration to minimize carry-over between analyses. A typical ECD chromatogram depicting TCE and TeCB peaks is shown in Figure 4.1.

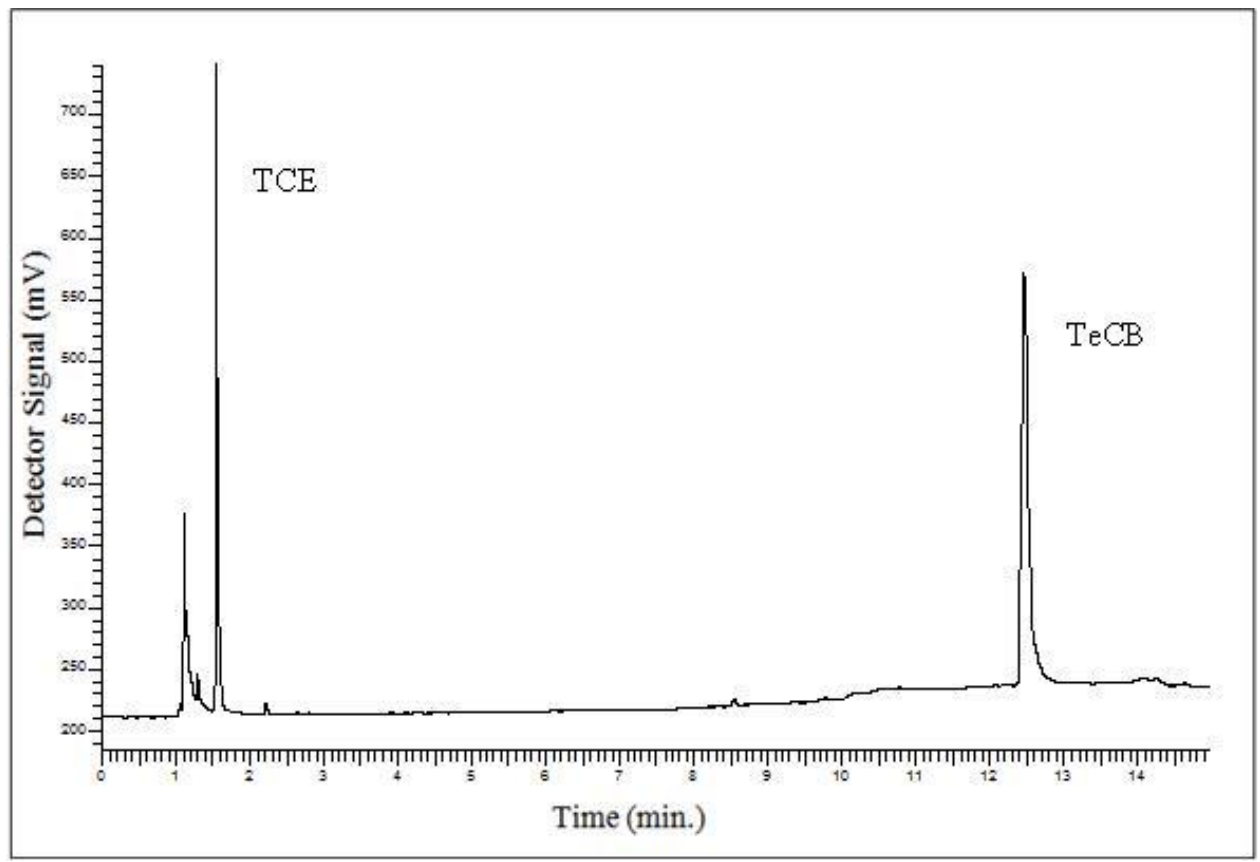

Figure 4.1. ECD Chromatogram Depicting TCE and TeCB Peaks

In Figure 4.1, trichloroethylene (TCE) and 1,2,4,5-tetrachlorobenzene (TeCB) eluted at approximately 1.5 minutes and 12.4 minutes, respectively. Slight baseline drift was observed for some experiments, but did not hinder the quantification of peak areas or the calibration of the instrument. During the course of this research, a new ECD anode and column were installed. After the new anode was installed, the sensitivity of the detector was altered, which required the development of a new gas chromatographic method. Both of the methods used to analyze TeCB are presented in Appendix A.

\subsubsection{TeCB Calibration Plot Development}

Calibration plots were developed to quantify unknown concentrations of TeCB in mixtures of water and ethanol. Peak area ratios (TeCB:TCE) were plotted against known TeCB 
concentrations in the standards. The peak area ratio was utilized so that variations in injected sample volumes would be corrected by the TCE peak area, which should theoretically be constant across experiments in the absence of autosampler error. A linear trendline with an intercept at the origin was added to the calibration plots, since a zero concentration of TeCB should theoretically yield a zero peak area. The slope of the trendline was used to quantify unknown concentrations of TeCB samples from batch reactor experiments. A representative TeCB calibration plot is presented in Figure 4.2.

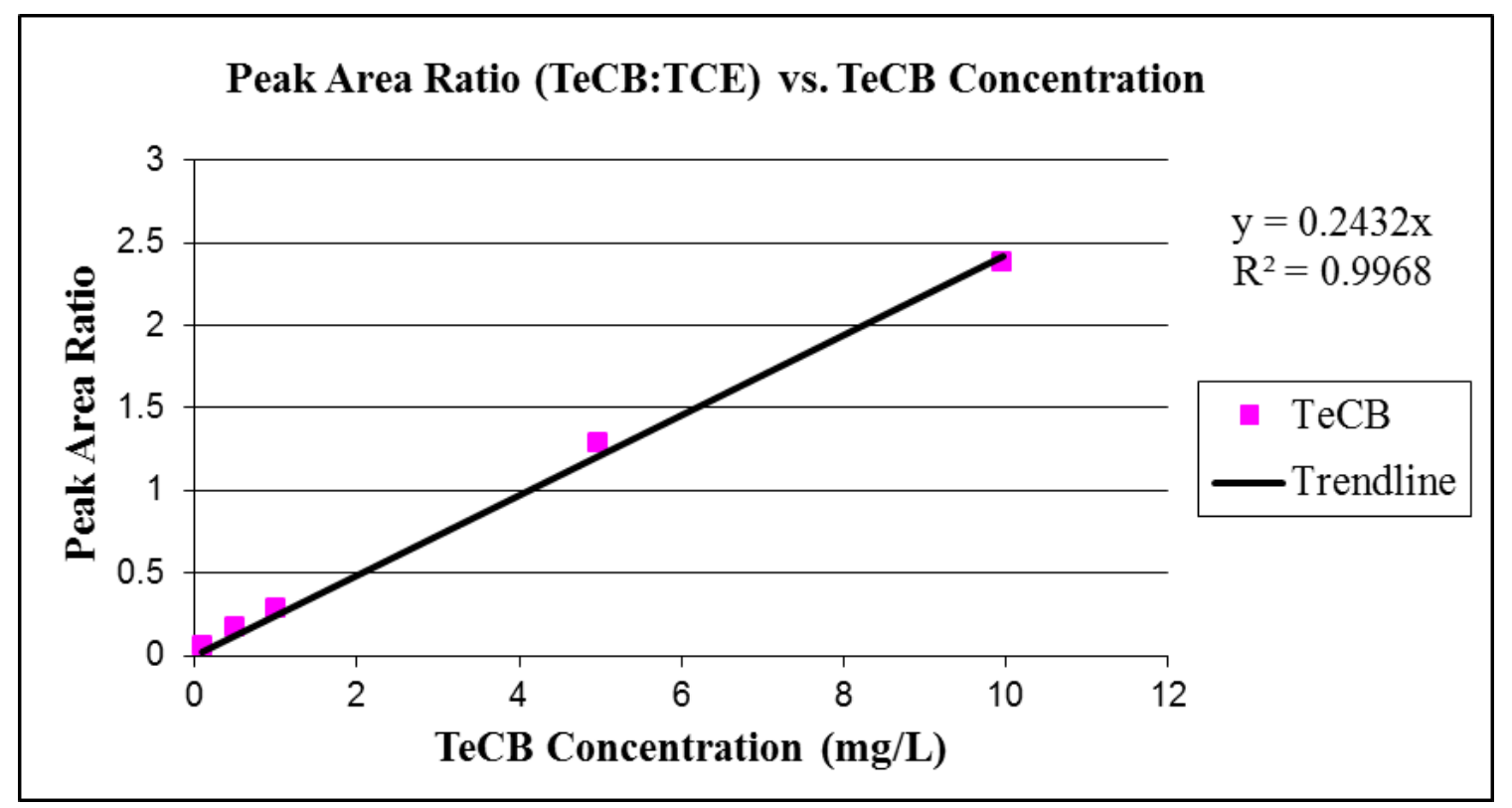

Figure 4.2. Calibration Plot for TeCB in 50:50 Water/Ethanol Solvent (Vol:Vol, Before Mixing)

\subsection{Preparation and Analysis of Cyclohexane and Benzene}

\subsubsection{Preparation of Stock Solutions and Standards}

Unlike TeCB, cyclohexane and benzene are not electronegative chemical species and cannot be detected by the ECD. Thus, cyclohexane and benzene were quantified using the FID. Combined cyclohexane and benzene stock solutions were used to create standards for GC calibration, since cyclohexane and benzene are often both present in the batch reactor solvent. 
Stock solutions of $1550 \mathrm{mg} / \mathrm{L}$ cyclohexane and $1746 \mathrm{mg} / \mathrm{L}$ benzene were prepared by adding $200 \mu \mathrm{L}$ of cyclohexane and $200 \mu \mathrm{L}$ of benzene to $100 \mathrm{~mL}$ of ethanol. Serial dilutions were then performed to achieve standards of known concentrations of approximately $0.112,0.56,1.12,5.6$, and $11.2 \mathrm{mg} / \mathrm{L}$ benzene and 0.0992, 0.496, 0.992, 4.96, and $9.92 \mathrm{mg} / \mathrm{L}$ cyclohexane in mixtures of water and ethanol. Standards were stored in the freezer in $20 \mathrm{~mL}$ borosilicate glass vials with screw caps with PTFE-lined septa to minimize volatilization. Any remaining stock was stored in the freezer in a $250 \mathrm{~mL}$ glass vial affixed with a glass cap. In accordance with the guidelines developed by Osborn (2011), the solutions were stored for a maximum of one week.

The combined cyclohexane and benzene standards were prepared for GC/FID analysis by placing $2 \mathrm{~mL}$ of each standard in two $5 \mathrm{~mL}$ borosilicate glass vials affixed with open screw caps with PTFE-lined septa. The resulting ten sample vials were shaken on a VWR OS-500 Orbital Shaker at approximately $200 \mathrm{rpm}$ for one hour. The samples were then equilibrated with the vial headspace (i.e. allowed to rest undisturbed) for 24 hours. Then, $1.0 \mathrm{~mL}$ of headspace was withdrawn with a gas-tight syringe and manually injected into the GC.

\subsubsection{FID Analysis of Cyclohexane and Benzene}

Ethanol was used as an internal standard in the FID analysis of cyclohexane and benzene, since it comprises a known fraction of the solvent and theoretically should be constant for experiments conducted with the same water/ethanol solvent composition. The mid-polarity RTX1301 column recommended by Osborn (2011) to achieve separation of ethanol, cyclohexane, and benzene was used for FID analysis (Table 4.2). The gas chromatograph analysis methods used for the analysis of benzene and cyclohexane were modeled after the work of Osborn (2011), and are detailed in Appendix B. A low carrier gas (i.e. helium) flow rate of $3 \mathrm{~mL} / \mathrm{min}$ was used to achieve separation of cyclohexane and benzene and a timed split event (SPL1) at 0.5 minutes 
was used to conserve gas between runs. Injection of headspace was performed manually using a $1000 \mu \mathrm{L}$ gas-tight Hamilton syringe. Extracted standards were analyzed in the order of least to most concentrated solutions to minimize column carry-over between analyses. A typical FID chromatogram is shown in Figure 3.3.

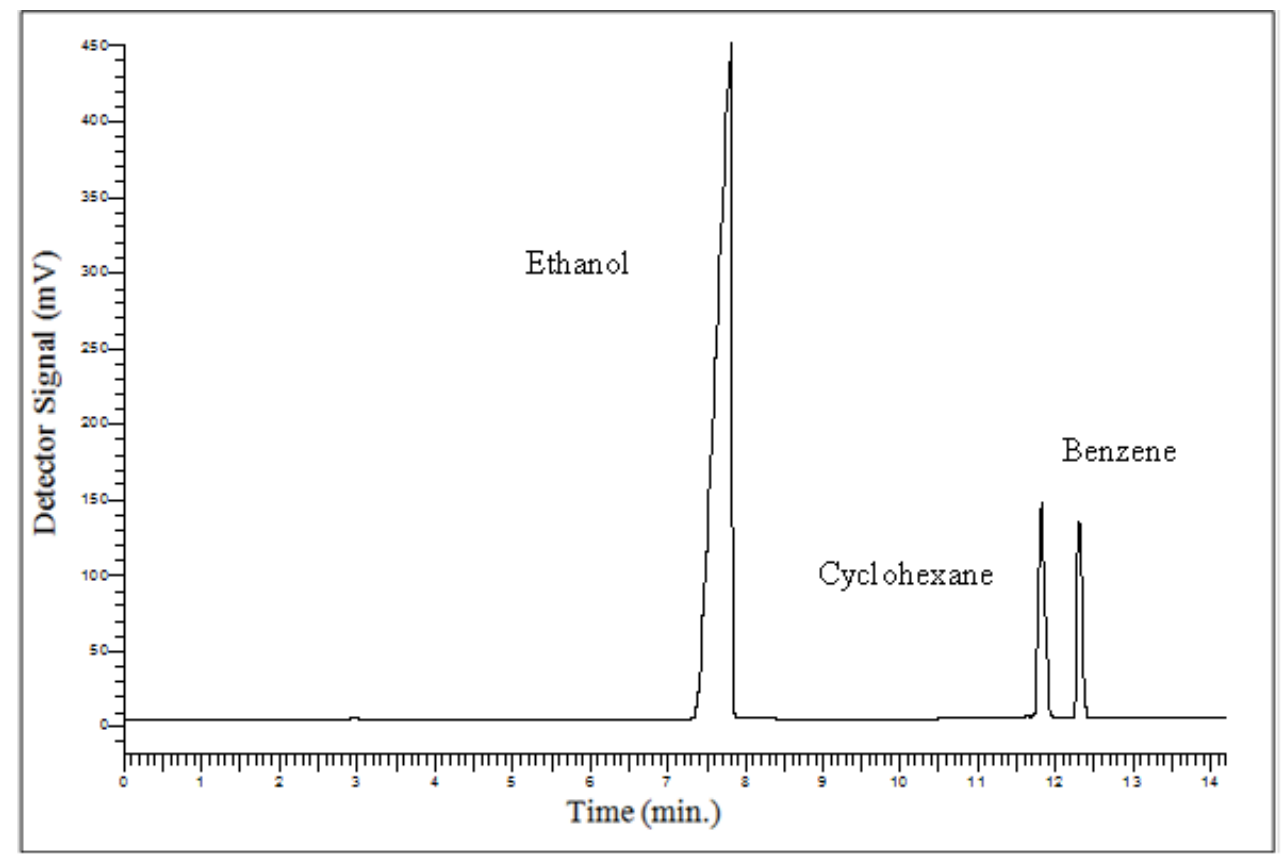

Figure 4.3. FID Chromatogram Depicting Ethanol, Cyclohexane, and Benzene Peaks

\subsubsection{Cyclohexane and Benzene Calibration Plot Development}

Calibration plots were developed to quantify unknown concentrations of cyclohexane and benzene in water-and-ethanol solvents. The peak area ratios of cyclohexane-to-ethanol and benzene-to-ethanol were plotted against known cyclohexane and benzene concentrations in the standards. A peak area ratio was used for the calibration so that any variations in the manually injected sample volumes would be corrected by the internal standard. A linear trendline with an intercept at the origin was added to the calibration plots, since a zero concentration of cyclohexane or benzene theoretically should produce no signal and thus yield a zero peak area. A 
representative cyclohexane calibration plot is shown in Figure 4.4, and a representative benzene calibration plot is shown in Figure 3.5.

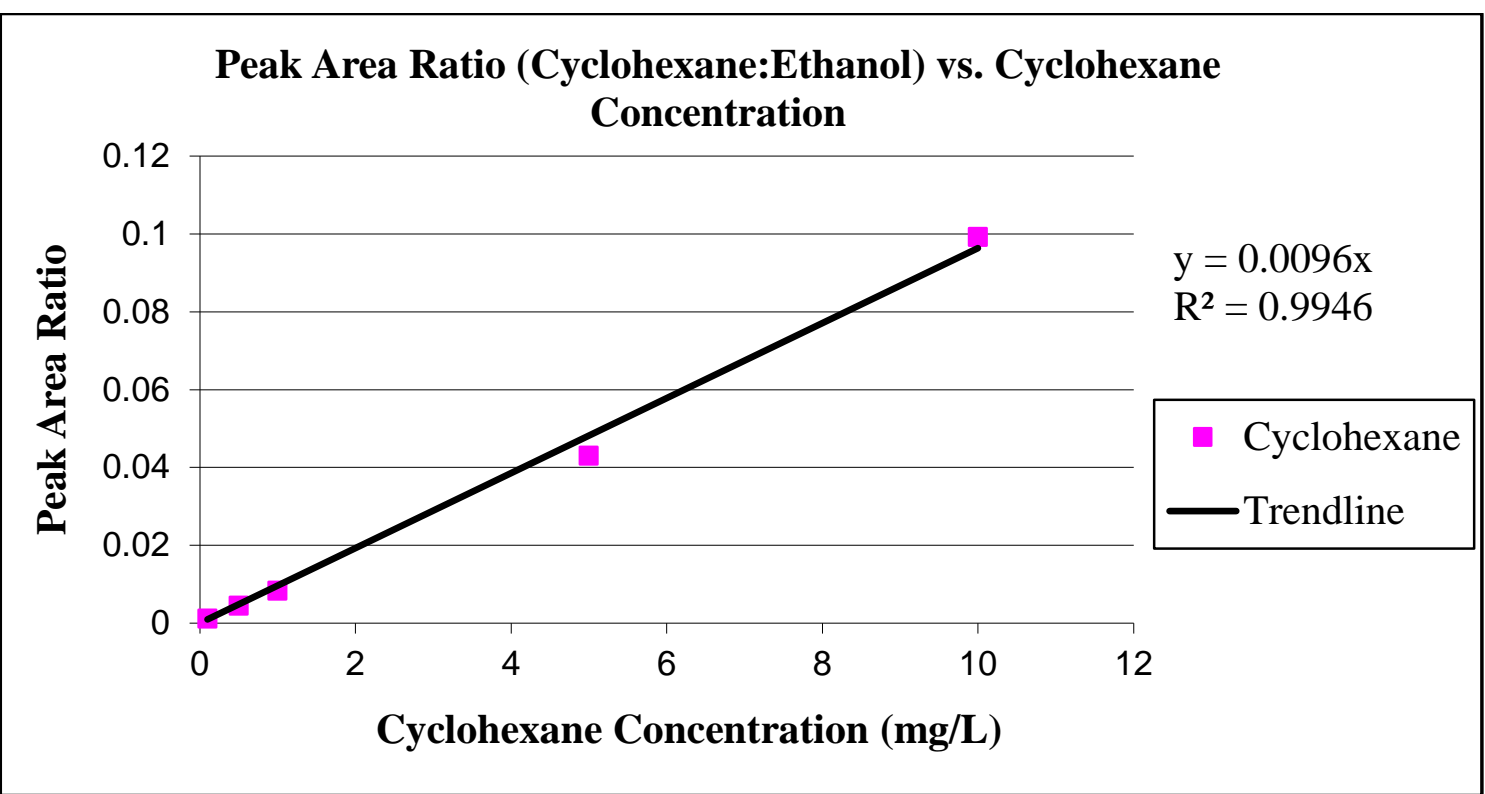

Figure 4.4. Calibration Plot for Cyclohexane in 33:67 Water/Ethanol Solvent (Vol:Vol, Before Mixing)

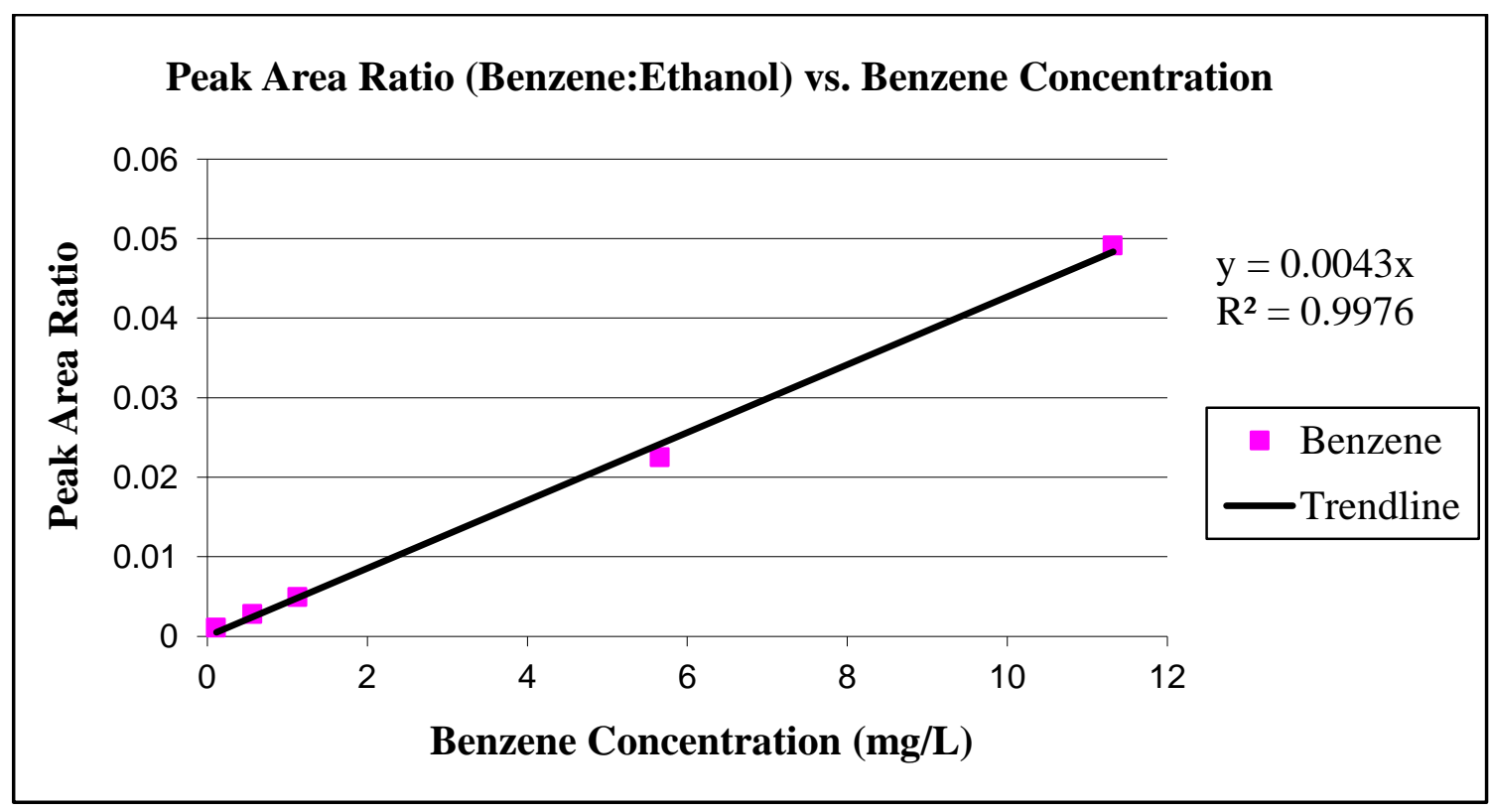

Figure 4.5. Calibration Plot for Benzene in 33:67 Water/Ethanol Solvent (Vol:Vol, Before Mixing) 


\subsection{Batch Reactor Experiments}

\subsubsection{Batch Reactor General Procedure}

All batch reactor experiments were conducted with a Parr 3911 hydrogenation apparatus, which was operated within a fume hood. The apparatus included a bottle rocker, a reaction bottle pressure gauge, a $4 \mathrm{~L}$ hydrogen gas tank, and a gas tank pressure gauge. The $4 \mathrm{~L}$ hydrogen gas tank allowed monitoring of hydrogen gas pressure in the reaction bottle. The hydrogen gas tank pressure gauge was connected to a hydrogen gas cylinder. Catalytic reactions took place inside a $500 \mathrm{~mL}$ borosilicate glass reaction bottle, which was sealed with a rubber stopper and connected to the hydrogen gas tank with polypropylene tubing. The reaction bottle had a maximum pressure limit of $60 \mathrm{psi}$, and was surrounded by a metal bottle shield during experiments for safety reasons. The reaction bottle was agitated at $200 \mathrm{rpm}$ on the apparatus' bottle rocker during experiments to minimize potential mass transfer limitations (Smith and Notheisz 1999).

All experiments were conducted by filling the reaction bottle with $300 \mathrm{~mL}$ of solvent in the desired ratio of water to ethanol. For example, to achieve a 50:50 water/ethanol solvent composition, $150 \mathrm{~mL}$ of water and $150 \mathrm{~mL}$ of ethanol were added to the reaction bottle. The resulting volume of the solvent may have been slightly less than $300 \mathrm{~mL}$ due to the negative volume effect of mixing water and ethanol. However, any volume change due to this effect would be slight and can be considered negligible (Wee and Cunningham 2008).

The solvent was then spiked with $10 \mathrm{mg} / \mathrm{L} \mathrm{TeCB}$ using the TeCB stock solution described in Section 4.3.1. Two samples of the spiked solvent were analyzed using the ECD to confirm the initial concentration of TeCB. Then, approximately $0.2 \mathrm{~g}$ of Pd-on- $\mathrm{Al}_{2} \mathrm{O}_{3}$ catalyst and $0.9 \mathrm{~g}$ of $\mathrm{Rh}-\mathrm{on}-\mathrm{Al}_{2} \mathrm{O}_{3}$ catalyst were added to the reaction bottle. Due to slight variations in 
the mass of individual catalyst pellets, the added $\mathrm{Pd}$-on- $\mathrm{Al}_{2} \mathrm{O}_{3}$ catalyst mass varied from $0.1986 \mathrm{~g}$ to $0.2171 \mathrm{~g}$ and the added $\mathrm{Rh}-\mathrm{on}-\mathrm{Al}_{2} \mathrm{O}_{3}$ catalyst mass varied from $0.9017 \mathrm{~g}$ to $0.9390 \mathrm{~g}$.

After the reaction bottle was connected to the Parr hydrogenation apparatus, any air in the bottle headspace was removed by filling the bottle with hydrogen gas to at least 45 psi and then venting the bottle headspace. The venting procedure was repeated three times to ensure that any air was fully evacuated. After venting, the reaction bottle was pressurized to the desired hydrogen pressure and the hydrogenation apparatus was activated. The reaction bottle remained open to the hydrogen tank during experiments to maintain a constant hydrogen pressure.

After the desired reaction time had elapsed, the reaction bottle was isolated from the hydrogen tank and the bottle headspace was vented. A $10 \mathrm{~mL}$ sample of the reaction bottle solvent was filtered through a Whatman 934-AH glass microfiber filter ( $24 \mathrm{~mm}$ diameter) by syringe. Two aliquots $(2 \mathrm{~mL}$ each) from the $10 \mathrm{~mL}$ sample were tested for final concentrations of TeCB using the methods described in Section 4.3, and two aliquots ( $2 \mathrm{~mL}$ each) were tested for final concentrations of benzene and cyclohexane according to the methods described in Section 4.4. The rubber stopper and tubing used to connect the reaction bottle to the hydrogenation apparatus were cleaned after each experiment. Fresh catalyst was used for each experiment to minimize the potential influence of catalyst deactivation on catalytic reaction rates.

\subsubsection{Experimental Design}

In order to investigate the effects of solvent composition and hydrogen pressure on HDH and hydrogenation reaction rates, batch experiments were conducted at multiple reaction times under the following operating conditions: (1) 50:50 water/ethanol solvent and 50 psi; (2) 67:33 water/ethanol solvent and 50 psi; (3) 33:67 water/ethanol solvent and 50 psi; (4) 50:50 water/ethanol solvent and $30 \mathrm{psi}$; and (5) 50:50 water/ethanol solvent and 10 psi. Thus, catalytic 
reaction rates were investigated for three different water/ethanol solvent compositions (50:50, 67:33, 33:67) and three different hydrogen pressures (50 psi, 30 psi, 10 psi). Higher water-toethanol ratios were not investigated since Wee and Cunningham (2013) previously found that efficiency of TeCB extraction from soil declines for solvent compositions of $67 \%$ water or higher. Hydrogen pressure experiments were conducted with a 50:50 water/ethanol solvent, since this solvent composition has been successfully used to treat contaminated soil in the semicontinuous REACH system (Wee and Cunningham 2011). For all experiments, the sampling and hydrogenation apparatus operating procedures described in Section 4.5.1 were followed. Table 4.3 details the run times and gas chromatographic methods used for each set of experimental conditions. All experiments were performed in duplicate. One additional experiment was conducted with a 50:50 water/ethanol solvent composition at 5 psi hydrogen pressure to determine if the reactions would still occur at a lower hydrogen pressure.

Two different GC/ECD methods were used during the course of the experiments due to the DB-WAX column being damaged during maintenance. Two different GC/FID methods were also used. The FID method was altered to achieve better separation of benzene and cyclohexane. All GC methods are described in Appendices A and B.

Table 4.3. Batch Experiment Run Times and Gas Chromatographic Methods

\begin{tabular}{|c|c|c|}
\hline Experimental Conditions & Run Times (hr) & Gas Chromatographic Methods \\
\hline $50: 50$ water/ethanol and $50 \mathrm{psi}$ & $1,2,4,6,8,12$ & ECD Method 1 / FID Method 1 \\
\hline $67: 33$ water/ethanol and 50 psi & $0.25,0.5,0.75,1,1.5$ & ECD Method 2 / FID Method 2 \\
\hline $33: 67$ water/ethanol and $50 \mathrm{psi}$ & $1.5,4,8,12,18$ & ECD Method 2 / FID Method 2 \\
\hline 50:50 water/ethanol and $30 \mathrm{psi}$ & $1,2,4,8,12$ & $\begin{array}{c}\text { ECD Method } 1 \text { ( } 8 \mathrm{hr} \text { experiments) } \\
\text { ECD Method } 2(1,2,4,12 \mathrm{hr}) \\
\text { FID Method } 2\end{array}$ \\
\hline 50:50 water/ethanol and $10 \mathrm{psi}$ & $1,2,4,8,12$ & ECD Method 2 / FID Method 2 \\
\hline
\end{tabular}

Note: ECD methods can be found in Appendix A, and FID methods can be found in Appendix B. 


\subsubsection{Control Experiments}

A control experiment was designed to test for alternative sources of contaminant mass loss other than catalytic reactions, such as volatilization or sorption onto the catalysts' surfaces. The experiment was conducted in duplicate at intermediate reaction conditions (50:50 water/ethanol solvent composition and $30 \mathrm{psi}$ ). The control experiments were carried out in accordance with the general experimental method detailed in Section 4.5.1. However, hydrogen gas was replaced by nitrogen gas to ensure that no catalytic HDH or hydrogenation reactions would occur during control experiments.

For these experiments, the reaction bottle was spiked with approximately $10 \mathrm{mg} / \mathrm{L}$ each of cyclohexane, benzene, and TeCB in $150 \mathrm{~mL}$ of water and $150 \mathrm{~mL}$ of ethanol. Four initial samples were taken. Two were analyzed for initial TeCB concentration using the methods described in Section 4.3, and two were analyzed for initial cyclohexane and benzene concentrations using the methods described in Section 4.4. The reactor was then shaken under 30 psi of $\mathrm{N}_{2}$ pressure. After eight hours, the reactor was stopped and four final samples were taken. Two samples were analyzed for resultant concentrations of TeCB, and two samples were analyzed for resultant concentrations of cyclohexane and benzene. Finally, the ratio of final concentrations to initial concentrations was calculated to determine the mass percentage lost for each chemical.

\subsection{Lessons Learned}

Some general lessons can be learned from the research conducted for this thesis. The first is that it is crucial to establish a regular maintenance schedule for gas chromatographs to obtain consistent and reproducible results. In addition to regular maintenance visits by a skilled technician, regular maintenance activities should include the replacement of injector cap septa, 
which can core over time. Septa coring can disrupt automatic gas pressure and flow rate regulation. For this research, the relatively wide-gauge needle used to manually inject $1 \mathrm{~mL}$ of headspace for FID analysis of cyclohexane and benzene required more frequent septa replacement than autosampler injections.

Secondly, chemical solubilities should be considered when developing a stock solution preparation method. For this research, benzene and cyclohexane stock solutions were initially prepared in water-and-ethanol mixtures. However, the relatively low solubility of benzene and cyclohexane in water led to inaccurate standards and calibration curves. It was determined to be necessary to prepare benzene and cyclohexane stock solutions in pure ethanol.

Thirdly, care should be taken when preparing solutions of volatile or semi-volatile chemicals with small volume (e.g. $10 \mu \mathrm{L})$ pipette tips. For this research, more consistent calibration curves were obtained when larger volumes of cyclohexane and benzene were added to stock solutions. Stock solutions of approximately $389 \mathrm{mg} / \mathrm{L}$ cyclohexane and $438 \mathrm{mg} / \mathrm{L}$ benzene were initially prepared in a $20 \mathrm{~mL}$ borosilicate glass vial by adding $10 \mu \mathrm{L}$ of cyclohexane and $10 \mu \mathrm{L}$ of benzene to $20 \mathrm{~mL}$ of ethanol. However, inconsistent results were obtained using this stock preparation method.

For experiments conducted at 33:67 water/ethanol solvent composition and 50 psi, the original calibration curve was determined to be inaccurate because it suggested that over twice the initial measured TeCB concentration was converted to cyclohexane. Therefore, a new calibration curve was prepared using the stock preparation method described in Section 4.4.1, where $200 \mu \mathrm{L}$ of cyclohexane and $200 \mu \mathrm{L}$ of benzene were added to $100 \mathrm{~mL}$ of ethanol. The new calibration curve was used to quantify cyclohexane and benzene concentrations. For all other experimental conditions, the calibrations were determined to be reasonable and were not altered. 


\section{CHAPTER 5: RESULTS AND DISCUSSION}

In Chapter 1 it was hypothesized that the kinetics of the catalytic conversion of 1,2,4,5tetrachlorobenzene (TeCB) to cyclohexane were influenced by solvent composition and hydrogen pressure. Specifically, an increased ratio of water to ethanol in the solvent was hypothesized to increase the reaction rates of both Pd-catalyzed hydrodehalogenation $(\mathrm{HDH})$ and Rh-catalyzed hydrogenation. It was also hypothesized that catalytic reaction rates would be constant above a hydrogen pressure threshold, but would decrease with decreased hydrogen pressure below the threshold. The conceptual basis of these hypotheses is explained in Chapter 3 .

The effects of solvent composition and hydrogen pressure on the catalytic conversion of TeCB to cyclohexane were investigated by varying water/ethanol solvent compositions and hydrogen pressures in a series of batch reactor experiments. Three sets of experiments were conducted at $50 \mathrm{psi}, 30 \mathrm{psi}$, and $10 \mathrm{psi}$ hydrogen pressures while holding solvent composition constant at 50:50 water/ethanol. Two additional sets of experiments were conducted at 67:33 and 33:67 water/ethanol solvent compositions while holding hydrogen pressure constant at $50 \mathrm{psi}$. All water-to-ethanol solvent composition ratios are reported by volume before mixing.

The objectives of this section are to present and discuss the findings of the batch reactor experiments in the context of prior research on the effects of solvent composition and hydrogen pressure on catalytic $\mathrm{HDH}$ and hydrogenation reaction kinetics. The process used to estimate the $\mathrm{HDH}$ and hydrogenation reaction rate coefficients will be presented in Section 5.1. The results of control experiments will be presented in Section 5.2. The effects of solvent composition and 
hydrogen pressure will be discussed in Section 5.3 and Section 5.4, respectively. Finally, the application of these findings to the REACH technology will be discussed in Section 5.5.

\subsection{Estimation of Reaction Rate Coefficients}

The apparent first order kinetic model developed in Chapter 3 was used to estimate HDH and hydrogenation reaction rate coefficients for each set of experimental conditions. As described in Section 2.1, the catalytic conversion of TeCB to cyclohexane occurs via two reactions in series - the Pd-catalyzed $\mathrm{HDH}$ of TeCB to benzene and the $\mathrm{Rh}$-catalyzed hydrogenation of benzene to cyclohexane. Recall the following mass balances for the concentrations of $\mathrm{TeCB}$, benzene, and cyclohexane in a batch reactor:

$$
\begin{gathered}
\frac{d C_{T}}{d t}=-k_{1} C_{T} \\
\frac{d C_{B}}{d t}=k_{1} C_{T}-k_{2} C_{B} \\
\frac{d C_{C}}{d t}=k_{2} C_{B}
\end{gathered}
$$

where $k_{1}$ is the apparent HDH rate coefficient, $k_{2}$ is the apparent hydrogenation rate coefficient, $C_{T}$ is the liquid-phase TeCB concentration, $C_{B}$ is the liquid-phase benzene concentration, and $C_{C}$ is the liquid-phase cyclohexane concentration.

Assuming only TeCB is initially present in the reactor, equations (13), (21), and (22) can be solved to obtain the following solved mass balances, which were previously employed by Osborn (2011) to obtain estimates for HDH and hydrogenation rate coefficients:

$$
\begin{gathered}
C_{T}=C_{0} e^{-k_{1} t} \\
C_{B}=\frac{k_{1}}{k_{2}-k_{1}} C_{0}\left(e^{-k_{1} t}-e^{-k_{2} t}\right)
\end{gathered}
$$




$$
C_{C}=C_{0}\left(\frac{k_{1}}{k_{2}-k_{1}} e^{-k_{2} t}-\frac{k_{2}}{k_{2}-k_{1}} e^{-k_{1} t}+1\right)
$$

where $C_{0}$ is the initial liquid-phase TeCB concentration and $t$ is time.

Equations (23) - (25) are based on the mass balances developed in Chapter 3, which are based on the assumption that no mass enters or exits the batch reactor. While the mass balances account for changes in liquid-phase chemical concentrations due to catalytic HDH and hydrogenation reactions and sorption to the catalyst surface, they do not account for potential mass loss due to volatilization. They also do not isolate the potential influence of catalyst deactivation on reaction rates either from poisoning by chloride ions released during HDH or from exposure to air during storage. Wee and Cunningham (2008) previously saw a gradual loss of Pd-on- $\mathrm{Al}_{2} \mathrm{O}_{3}$ catalyst activity over a period of less than one year, which was attributed to exposure to air during storage. For this thesis, experiments were conducted over a 10 month period, during which time the same batch of $\mathrm{Pd}$-on- $\mathrm{Al}_{2} \mathrm{O}_{3}$ catalyst was used. Three separate containers of $\mathrm{Rh}-\mathrm{on}-\mathrm{Al}_{2} \mathrm{O}_{3}$ catalyst were used over the course of this research, though all catalysts were purchased simultaneously.

The solved mass balances provide a simple means of estimating apparent HDH and hydrogenation reaction rate coefficients. The reaction rate coefficients for different experimental conditions are compared in Section 5.3 for varying solvent compositions and in Section 5.4 for varying hydrogen pressures. The best-fit values for $k_{1}$ and $k_{2}$ and the $95 \%$ confidence intervals for these values were obtained using the fit function in Matlab, which performs a nonlinear least squares regression. Equation (23) was fitted to the observed TeCB data to obtain estimates for $\mathrm{HDH}$ rate coefficients ( $k_{1}$ values). Equation (24) was then fitted to the observed benzene data using the $k_{1}$ values to estimate hydrogenation rate coefficients ( $k_{2}$ values). The best-fit values of $k_{1}$ and $k_{2}$ can be used to predict $C_{T}, C_{B}$, and $C_{C}$ according to equations (23) - (25). 
As explained in Chapter 3, each experiment was designed to start with an initial TeCB concentration of $10 \mathrm{mg} / \mathrm{L}$. However, it is possible that experimental errors may have caused $C_{0}$ to deviate slightly from the target of $10 \mathrm{mg} / \mathrm{L}$. Therefore, $C_{0}$ was measured at the start of each experiment after TeCB addition to the solvent. Due to the potential dependence of reaction rates on initial TeCB concentration, the average initial TeCB concentrations for each set of experiments are listed in Table 5.1. Additionally, because of the potential for mass loss due to volatilization or sorption to the catalyst surface, the percentages of initial TeCB mass recovered for each set of experimental conditions are also presented in Table 5.1.

Table 5.1. Average Initial TeCB Concentrations and Mass Recovery

\begin{tabular}{|l|c|c|}
\hline Experimental Conditions & $\begin{array}{c}\text { Average Initial TeCB } \\
\text { Concentration }\left(\boldsymbol{C}_{\mathbf{0}}\right)\end{array}$ & $\begin{array}{c}\text { Percentage of Initial } \\
\text { TeCB Recovered }\end{array}$ \\
\hline 50:50 water/ethanol; $50 \mathrm{psi}$ & $10 \mathrm{mg} / \mathrm{L}$ & $79 \%$ \\
\hline 67:33 water/ethanol; $50 \mathrm{psi}$ & $10 \mathrm{mg} / \mathrm{L}$ & $94 \%$ \\
\hline 33:67 water/ethanol; $50 \mathrm{psi}$ & $9 \mathrm{mg} / \mathrm{L}$ & $101 \%$ \\
\hline 50:50 water/ethanol; $30 \mathrm{psi}$ & $11 \mathrm{mg} / \mathrm{L}$ & $89 \%$ \\
\hline 50:50 water/ethanol; $10 \mathrm{psi}$ & $12 \mathrm{mg} / \mathrm{L}$ & $102 \%$ \\
\hline
\end{tabular}

While batch experiments were designed to have an initial concentration of $10 \mathrm{mg} / \mathrm{L}$, the measured initial TeCB concentrations ranged on average from 9-12 mg/L. Wee and Cunningham (2008) previously found that varying the initial TeCB concentration from 5-10 $\mathrm{mg} / \mathrm{L}$ did not significantly impact $\mathrm{HDH}$ reaction rates. Thus, the slight variations in initial TeCB concentration should not prohibit a comparison of estimated $\mathrm{HDH}$ and hydrogenation rate coefficients from different data sets. Mass recovery percentages slightly greater than $100 \%$ were observed for some data sets, which can be attributed to experimental error. Some variability in the average recovery of initial TeCB mass can also be observed in Table 5.1. However, the variability in the closure of the mass balance is consistent with the results of the control experiments presented 
subsequently in Section 5.2. Thus, variability in mass balance closure should not prohibit the comparison of reaction rate coefficients from different data sets.

\subsection{Control Experiments}

The overall high recovery percentage of initial TeCB mass measured during batch experiments shown in Table 5.1 suggests that losses of chemical mass to volatilization and sorption were not significant. However, mass recoveries lower than $75 \%$ were observed for a few individual experiments, which suggest that some mass loss did occur. The anomalously low average mass recovery of $79 \%$ for the initial set of experiments conducted at 50:50 water/ethanol compositions and 50 psi can partially be attributed to the use of a slow filtration method that was later modified to reduce the potential for volatilization while preparing samples for analysis.

Two control experiments were conducted as described in Section 4.5.3 at 30 psi nitrogen gas pressure and 50:50 water/ethanol solvent compositions to test for losses of TeCB, benzene, and cyclohexane due to volatilization or sorption to catalyst surfaces. The results of the control experiments were variable. Percentage losses of $0.28-2.3 \%$ for TeCB, $11-23 \%$ for benzene, and $23-47 \%$ for cyclohexane were observed after 8 hours in the batch reactor.

These results differed from the findings of Osborn (2011), who recorded 100\% loss of benzene, $20 \%$ loss of TeCB, and $0 \%$ loss of cyclohexane when conducting a control experiment with nitrogen gas and $\mathrm{Pd}$ and Rh catalysts in the same batch system. However, Osborn (2011) did not report the duration of the control experiment or the initial concentrations of the contaminants. Wee and Cunningham (2008) also conducted batch reactor control experiments using TeCB and Pd catalysts, and observed mostly insignificant TeCB losses due to sorption and volatilization. However, they found significant TeCB volatilization losses of $36-68 \%$ with a 67:33 water/ethanol solvent composition. For all other solvent compositions, they assumed that 
losses due to volatilization and sorption were minimal. In contrast to the findings of Wee and Cunningham (2008), significant mass losses were not observed for experiments conducted with a 67:33 water/ethanol solvent composition in this thesis.

Due to the high closure of the mass balance for most of the batch experimental results and the low observed losses of TeCB and benzene in the control experiments, it will be assumed that volatilization and sorption are minor loss mechanisms and that observed changes in TeCB and benzene concentrations can be used to estimate $\mathrm{HDH}$ and hydrogenation reaction rates.

\subsection{Effects of Solvent Composition}

Measured and predicted TeCB, benzene, and cyclohexane data are plotted in Figure 5.1, Figure 5.2, and Figure 5.3 for experiments conducted at 50 psi hydrogen pressure with 33:67, 50:50, and 67:33 water/ethanol solvents. Note that the time scale of the horizontal axis varies from 18 hours in Figure 5.1 to 12 hours in Figure 5.2 and 1.5 hours in Figure 5.3.

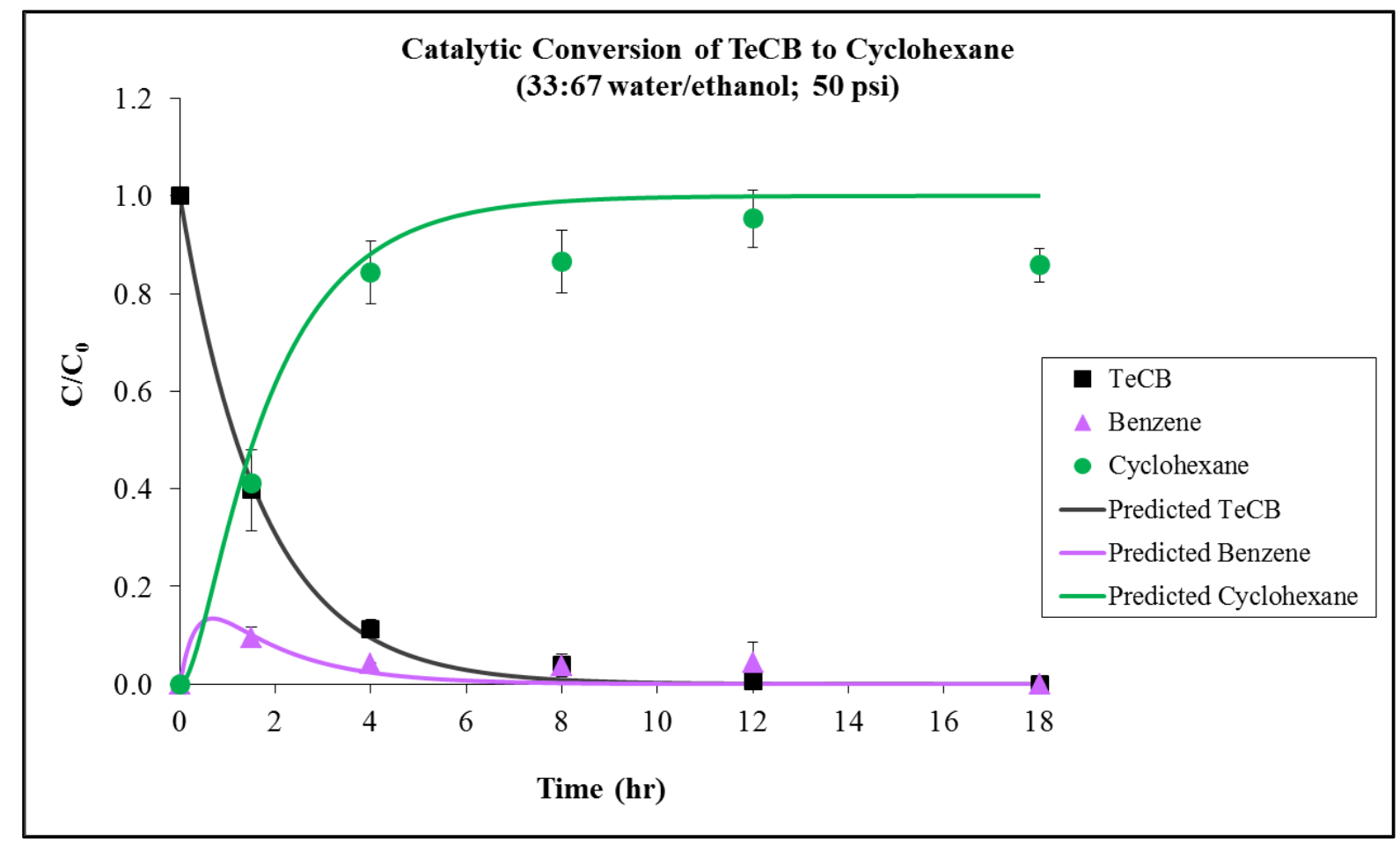

Figure 5.1. Batch Reactor Experiments for 33:67 Water/Ethanol Solvent Composition and 50 Psi Hydrogen Pressure 


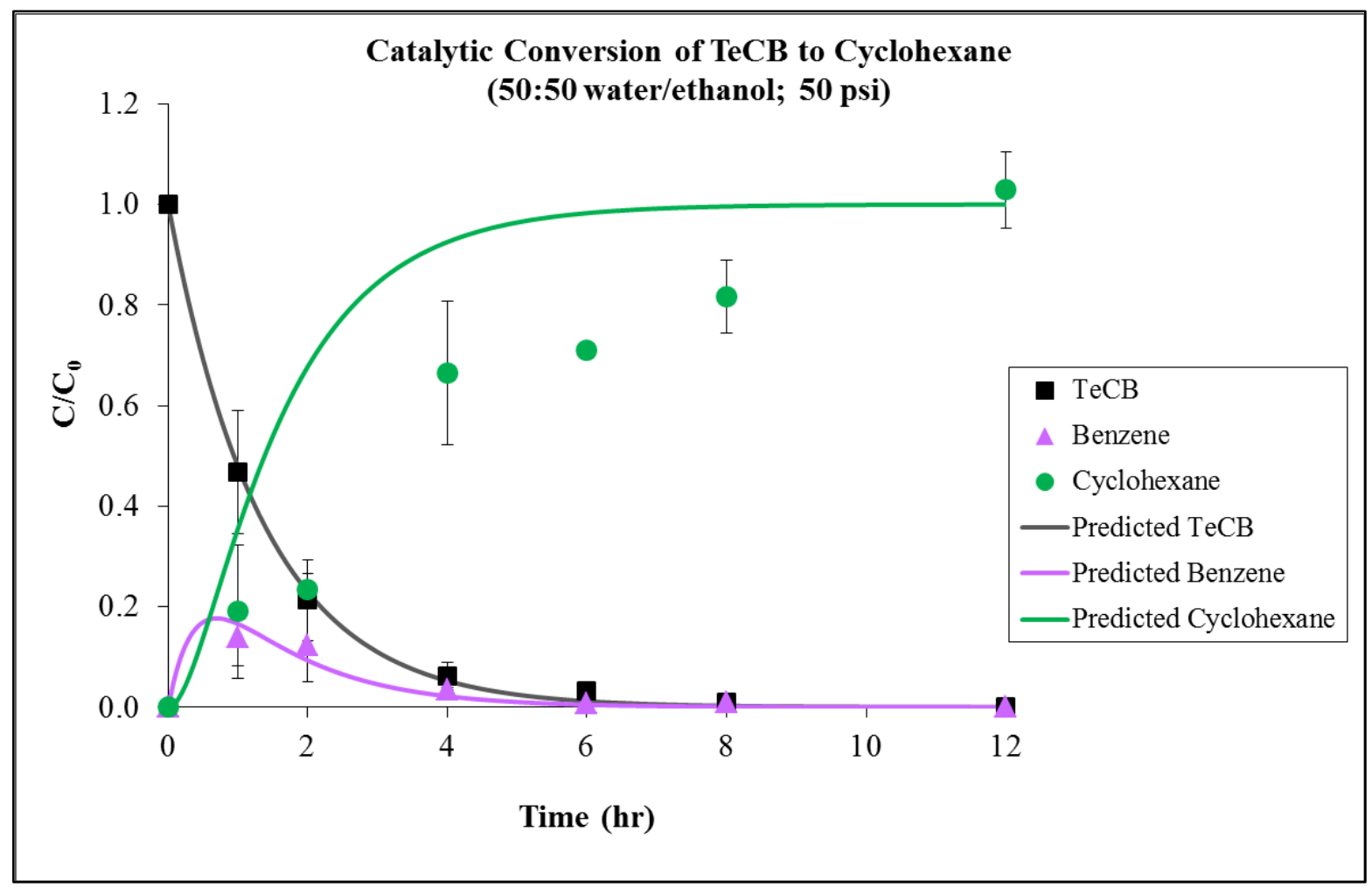

Figure 5.2. Batch Reactor Experiments for 50:50 Water/Ethanol Solvent Composition and 50 Psi Hydrogen Pressure

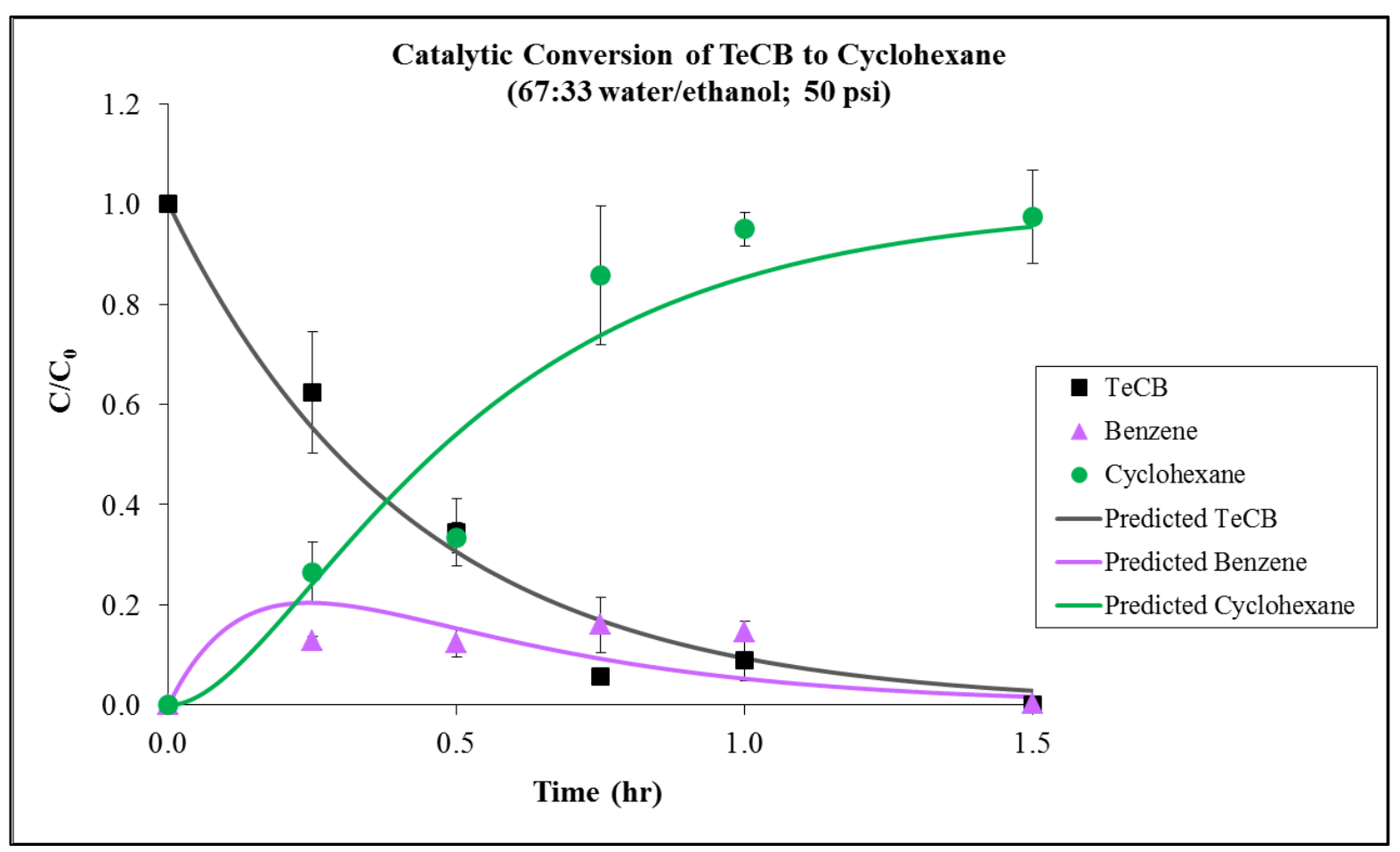

Figure 5.3. Batch Reactor Experiments for 67:33 Water/Ethanol Solvent Composition and 50 Psi Hydrogen Pressure 
All experiments were performed in duplicate. The data point is the arithmetic mean, and the error bars indicate maximum and minimum values for the two experiments. The complete conversion of TeCB to cyclohexane was achieved in a single batch reactor with all three water/ethanol solvent compositions tested $(50: 50,67: 33,33: 67)$ at 50 psi hydrogen pressure. Though the predicted cyclohexane values for experiments conducted with a $50 \%$ water solvent at 50 psi (Figure 5.2) often overestimate the measured values, this can be partially attributed to the low percentage of initial TeCB mass recovery for this data set. As mentioned previously, after the first set of experiments, which were conducted with a $50 \%$ water solvent at 50 psi, a more efficient filtration method was developed that resulted in a greater closure of the mass balance in subsequent experiments (Table 5.1).

The estimated reaction rate coefficients and reaction completion times for the three sets of solvent composition batch experiments are summarized in Table 5.2. The estimated time for complete conversion of TeCB to cyclohexane is defined as the required time to reduce TeCB and benzene concentrations below detectable limits $(0.1 \mathrm{mg} / \mathrm{L})$.

Table 5.2. Estimated Rate Coefficients and Reaction Times for Solvent Composition Experiments

\begin{tabular}{|l|c|c|c|}
\hline Experimental Conditions & $\begin{array}{c}\text { Apparent } \boldsymbol{k}_{\mathbf{1}} \\
\text { Reaction Rate } \\
\text { Coefficient } \\
\mathbf{( h r}^{-\mathbf{1}} \mathbf{)}\end{array}$ & $\begin{array}{c}\text { Apparent } \boldsymbol{k}_{\mathbf{2}} \\
\text { Reaction Rate } \\
\text { Coefficient } \\
\mathbf{( h r}^{-\mathbf{1}} \mathbf{)}\end{array}$ & $\begin{array}{c}\text { Observed Time } \\
\text { for Complete } \\
\text { Conversion (hr) }\end{array}$ \\
\hline 33:67 water/ethanol; 50 psi & $0.59 \pm 0.06$ & $2.92 \pm 1.68$ & $12-18$ \\
\hline 50:50 water/ethanol; 50 psi & $0.74 \pm 0.04$ & $2.50 \pm 0.55$ & $8-12$ \\
\hline 67:33 water/ethanol; 50 psi & $2.37 \pm 0.62$ & $6.51 \pm 4.03$ & $1-1.5$ \\
\hline
\end{tabular}

From the findings of Wee and Cunningham (2008) and Ticknor (2012), it was expected that an increased ratio of water to ethanol in the solvent would increase both HDH and hydrogenation reaction rates. As expected, the apparent $\mathrm{HDH}$ reaction rate coefficients $\left(k_{1}\right.$ 
values) increased with an increasing fraction of water in the solvent. The estimated $k_{1}$ values were $0.59 \mathrm{hr}^{-1}$ for $33 \%$ water, $0.74 \mathrm{hr}^{-1}$ for $50 \%$ water, and $2.37 \mathrm{hr}^{-1}$ for $67 \%$ water solvents. A similar trend of increased HDH reaction rate coefficients with increased water fraction in the solvent was noted by Wee and Cunningham (2008) for the HDH of TeCB.

The hydrogenation reaction rate coefficients ( $k_{2}$ values) showed a similar trend to the $k_{1}$ values, though the variability in the data resulted in large statistical uncertainty, as indicated by the broad $95 \%$ confidence intervals for experiments conducted with 33\% water and $67 \%$ water solvents. The $k_{2}$ values were $2.92 \mathrm{hr}^{-1}$ for $33 \%$ water, $2.50 \mathrm{hr}^{-1}$ for $50 \%$ water, and $6.51 \mathrm{hr}^{-1}$ for $67 \%$ water solvents. Thus, a large increase in reaction rate coefficients was not observed until the fraction of water in the solvent was increased to $67 \%$. The estimated reaction rate coefficient at $67 \%$ water is the fastest of all the experimental conditions tested, which is consistent with the hypothesis that water addition facilitates faster catalytic reaction rates. However, the increase falls within the $95 \%$ confidence intervals for the $k_{2}$ value so it cannot be said with $95 \%$ certainty that the reaction rate coefficient significantly increased.

The slight decrease in the hydrogenation reaction rate coefficient when the water fraction in the solvent was increased from $33 \%$ to $50 \%$ was an unexpected result. However, this result was not corroborated by a corresponding decrease in the observed time for complete conversion of $\mathrm{TeCB}$ to cyclohexane. When the fraction of water was increased from $33 \%$ to $50 \%$, the time for complete conversion actually decreased from 12-18 hours to 8-12 hours. Thus, the apparent increase in the hydrogenation reaction rate coefficient is not believed to represent a significant finding. It is possible that faster deactivation of the Rh catalyst occurred in the 33\% water solvent compared to the $50 \%$ water solvent, which resulted in an extended hydrogenation reaction time. However, catalyst deactivation effects cannot clearly be isolated in the data. 
Interestingly, a greater $k_{2}$ value $\left(2.50 \mathrm{hr}^{-1}\right)$ was found in this study for experiments conducted at $50 \%$ water and 50 psi than the $k_{2}$ value reported by Osborn (2011) at the same conditions $(0.55$ $\left.\mathrm{hr}^{-1}\right)$. However, the observed time for complete conversion of TeCB to cyclohexane (8-12 hours) was approximately the same as that reported by Osborn (2011) at the same conditions (12 hours).

The required time for complete conversion of TeCB to cyclohexane clearly decreased as the fraction of water in the solvent increased. The conversion time dramatically decreased from approximately 8-12 hours to 1-1.5 hours when the water fraction in the solvent was increased from $50 \%$ to $67 \%$. Ticknor (2012) also noted a decrease in the time required for complete conversion of TeCB to cyclohexane from the 12 hours observed by Osborn (2011) at a solvent composition of $50 \%$ water to 45 minutes with a $67 \%$ water solvent composition. The data in this study suggest that the increased hydrogenation rate observed by Ticknor (2012) when the water fraction in a water/ethanol solvent was increased from $50 \%$ to $67 \%$ was due to a solvent effect.

As previously mentioned, the 12 hour reaction time observed by Osborn (2011) at 50\% water and 50 psi corresponds well with the conversion time observed in this study (8-12 hours), though Osborn (2011) reported different $k_{1}$ and $k_{2}$ values. Ticknor (2012) achieved a slightly faster conversion time with a $67 \%$ water solvent composition at 50 psi (45 minutes) than that observed in this study (1-1.5 hours). However, Ticknor (2012) utilized a powdered palladium catalyst instead of the pelletized $\mathrm{Pd}$-on- $\mathrm{Al}_{2} \mathrm{O}_{3}$ catalyst used both in this study and by Osborn (2011). With the powdered palladium catalyst, Ticknor (2012) found that TeCB was completely hydrodehalogenated in 5 minutes. The accelerated $\mathrm{HDH}$ rate likely resulted in a shorter time for complete conversion of TeCB to cyclohexane than was observed in this thesis.

In this study, increasing the water fraction in the solvent from $33 \%$ to $50 \%$ did not appear to have as large an effect on reaction rates or conversion times as increasing the water fraction 
from $50 \%$ to $67 \%$. A slight but statistically significant increase in $k_{1}$ values $\left(0.59 \mathrm{hr}^{-1}\right.$ to $\left.0.74 \mathrm{hr}^{-1}\right)$ and a slight decrease in $k_{2}$ values $\left(2.92 \mathrm{hr}^{-1}\right.$ to $\left.2.50 \mathrm{hr}^{-1}\right)$ were observed when the water fraction was increased from $33 \%$ to $50 \%$. A larger increase in reaction rate coefficients for both HDH and hydrogenation was observed when the fraction of water was increased from $50 \%$ to $67 \%$. The highest $k_{1}$ value $\left(2.37 \mathrm{hr}^{-1}\right)$ and $k_{2}$ value $\left(6.51 \mathrm{hr}^{-1}\right)$ out of all the sets of batch experiments conducted were obtained with a $67 \%$ water solvent at 50 psi.

As discussed in Section 2.7, most HDH research groups concur that the addition of water to an alcohol solvent improves HDH reaction rates (Hoke et al. 1992; Benítez and Del Angel 1999; Xia et al. 2003; Concibido et al. 2006, 2007; Pozan and Boz 2008; Wee and Cunningham 2008; Gómez-Quero et al. 2010a). However, some research groups have observed that HDH reaction rates do not increase monotonically with the fraction of water in the solvent (Xia et al. 2003; Concibido et al. 2006, 2007; Pozan and Boz 2008). Concibido et al. (2005) and Pozan and Boz (2008) actually observed a decrease in the $\mathrm{HDH}$ reaction rate when greater than $50 \%$ water by volume was used in a water/methanol solvent. However, Concibido et al. (2006) later found that this effect was unique to a water/methanol solvent.

The solvent composition data in this thesis correspond well with the findings of Concibido et al. (2006), who observed no significant increase in $\mathrm{HDH}$ rate until at least 50\% water by volume was added to a water/ethanol solvent in the HDH of tetrachloroethylene (PCE). Akpa et al. (2012) similarly observed a "threshold" effect where the hydrogenation rate for 2butanone over Ru-on-C increased abruptly when the water molar fraction of a water/isopropanol solvent was increased to $90 \%$. The general trend of increasing HDH reaction rates with increased water content in the solvent also corresponds well with prior research on the REACH system conducted by Wee and Cunningham (2008) and Ticknor (2012). As discussed in Section 3.4, it is 
the belief of this author that a greater fraction of water in the solvent results in better adsorption of hydrophobic TeCB and benzene to the catalyst surface and, therefore, faster reaction rates.

\subsection{Effects of Hydrogen Pressure}

Measured and predicted TeCB, benzene, and cyclohexane data are plotted in Figure 5.4 and Figure 5.5 for batch reactor experiments conducted with a 50:50 water/ethanol solvent composition at $30 \mathrm{psi}$ and 10 psi hydrogen pressures. All experiments were performed in duplicate. As described in Section 5.3, the data point represents the arithmetic mean, and the error bars indicate maximum and minimum values for the two experiments. The batch experiment results for a 50:50 water/ethanol solvent composition at 50 psi were previously presented in Figure 5.2.

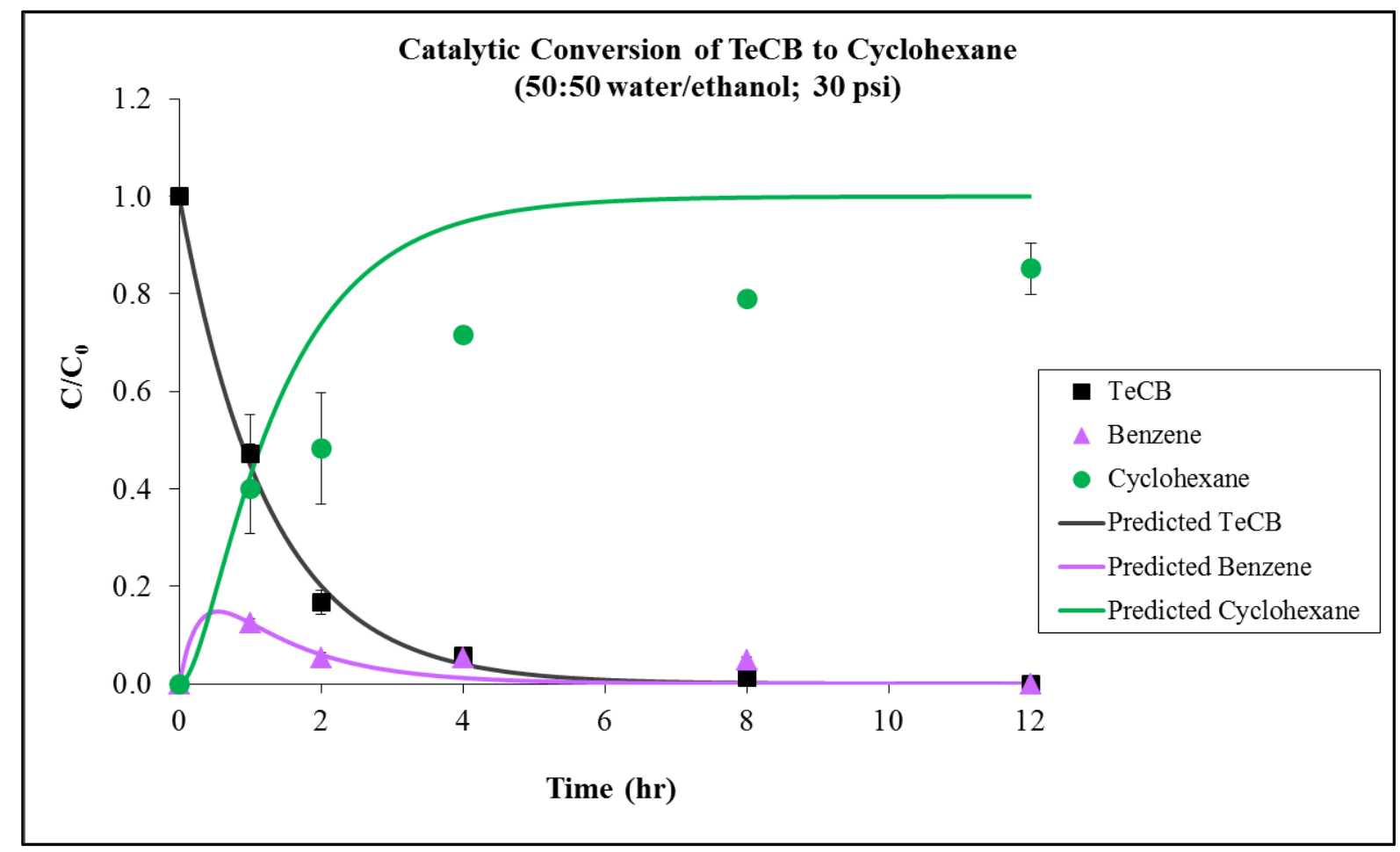

Figure 5.4. Batch Reactor Experiments for 50:50 Water/Ethanol Solvent Composition and 30 Psi Hydrogen Pressure 


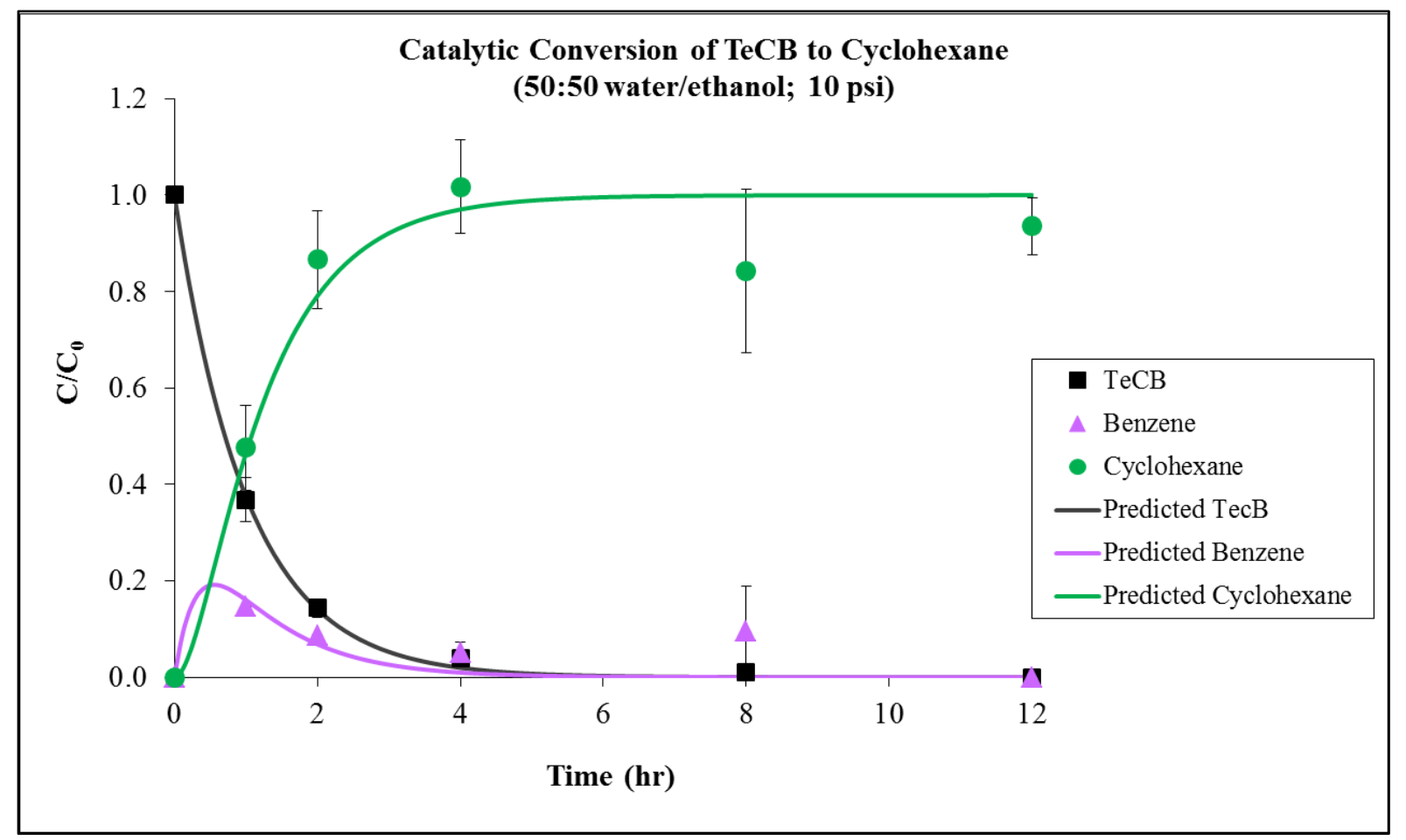

Figure 5.5. Batch Reactor Experiments for 50:50 Water/Ethanol Solvent Composition and 10 Psi Hydrogen Pressure

The complete conversion of TeCB to cyclohexane was achieved at all three hydrogen pressures (50 psi, $30 \mathrm{psi}, 10 \mathrm{psi}$ ) with a 50:50 water/ethanol solvent. The estimated reaction rate coefficients and reaction times for experiments conducted with a 50:50 water/ethanol solvent composition at $50 \mathrm{psi}, 30 \mathrm{psi}$, and $10 \mathrm{psi}$ are presented in Table 5.3. The predicted cyclohexane concentrations overestimate the measured cyclohexane concentrations in Figure 5.4 for batch experiments conducted at 30 psi hydrogen pressure. However, this can partially be attributed to the relatively low percentage of initial TeCB mass recovery for these experiments (89\%).

An additional eight-hour experiment was conducted at 5 psi hydrogen pressure to test if a minimum hydrogen pressure was required for the reactions to occur. Complete conversion of TeCB to cyclohexane was achieved after 8 hours. No observed conversion of TeCB occurred in control experiments when hydrogen gas was replaced by nitrogen, which suggests that a minimum hydrogen pressure threshold does exist. However, the minimum quantifiable pressure 
in the batch reactor is 3 psi. A different apparatus would likely be required to experimentally determine the hypothesized hydrogen pressure threshold, under which HDH and hydrogenation reaction rates are expected to decrease with decreasing hydrogen pressure.

Table 5.3. Estimated Rate Coefficients and Reaction Times for Hydrogen Pressure Experiments

\begin{tabular}{|l|c|c|c|}
\hline Experimental Conditions & $\begin{array}{c}\text { Apparent } \boldsymbol{k}_{\mathbf{1}} \\
\text { Reaction Rate } \\
\text { Coefficient } \\
\text { (hr }^{-\mathbf{1}} \text { ) }\end{array}$ & $\begin{array}{c}\text { Apparent } \boldsymbol{k}_{\mathbf{2}} \\
\text { Reaction Rate } \\
\text { Coefficient } \\
\mathbf{( h r}^{-\mathbf{1}} \text { ) }\end{array}$ & $\begin{array}{c}\text { Observed Time } \\
\text { for Complete } \\
\text { Conversion } \\
\text { (hr) }\end{array}$ \\
\hline 50:50 water/ethanol; 50 psi & $0.74 \pm 0.04$ & $2.50 \pm 0.55$ & $8-12$ \\
\hline 50:50 water/ethanol; 30 psi & $0.80 \pm 0.08$ & $3.49 \pm 1.67$ & $8-12$ \\
\hline 50:50 water/ethanol; $10 \mathrm{psi}$ & $0.98 \pm 0.05$ & $2.96 \pm 1.89$ & $8-12$ \\
\hline
\end{tabular}

Based on the Langmuir adsorption isotherm and the prior findings of Wee and Cunningham (2008), it was hypothesized that hydrogen pressure would have no effect on catalytic reaction rates above a certain threshold. Below this threshold, it was hypothesized that reaction rates would decrease with decreasing hydrogen pressure due to lower surface coverage of hydrogen on the catalyst. The data suggest a more complex hydrogen pressure effect.

Surprisingly, the HDH reaction rate coefficients ( $k_{1}$ values) increased monotonically with decreasing hydrogen pressures in the range of 10-50 psi, though the increase was not statistically significant with $95 \%$ confidence until the pressure was decreased from 30 psi to $10 \mathrm{psi}$. A slight but statistically significant increase in the HDH reaction rate coefficient was observed when the pressure was decreased from $30 \mathrm{psi}$ to $10 \mathrm{psi}\left(0.80 \mathrm{hr}^{-1}\right.$ to $\left.0.98 \mathrm{hr}^{-1}\right)$. Thus, it can be concluded with $95 \%$ confidence that $k_{1}$ was higher at 10 psi than at 30 psi or 50 psi. The $k_{1}$ values were similar for both $50 \mathrm{psi}$ and $30 \mathrm{psi}$ hydrogen pressures $\left(0.74 \mathrm{hr}^{-1}\right.$ vs. $\left.0.80 \mathrm{hr}^{-1}\right)$, with the difference between the values falling within their $95 \%$ confidence intervals. TeCB was observed to be completely converted to cyclohexane within 8-12 hours at all three hydrogen pressures. The 
similar observed conversion times suggest that the observed effect of hydrogen pressure on HDH rate coefficients is minor.

Since the data suggest that HDH reaction rate coefficients vary with hydrogen pressure, a power law estimate was obtained for the HDH reaction order with respect to hydrogen pressure. The estimate was obtained by plotting $k_{1}$ values against hydrogen pressure for experiments conducted with a 50:50 water/ethanol solvent and then utilizing Microsoft Excel's power law fit function. Based on this analysis, the HDH rate appears to be of order -0.18 with respect to hydrogen pressure.

Jujjuri and Keane (2010) similarly observed a slight increase in HDH reaction rates for chlorinated benzenes with decreasing hydrogen pressures in the range of approximately 0.68 13.6 psi. Jujjuri and Keane (2010) proposed that excess stoichiometric hydrogen due to increased hydrogen pressures can inhibit HDH reactions due to competitive adsorption of hydrogen and the displacement of intermediate products from the catalyst surface. In this thesis, it is possible that the observed $\mathrm{HDH}$ reaction rate increase when the hydrogen pressure was decreased from 30 to $10 \mathrm{psi}$ is indicative of a similar competitive adsorption effect between hydrogen and TeCB.

In contrast, some research groups have found hydrodechlorination reactions to be independent of hydrogen pressure (Kovenklioglu et al. 1992; Benítez and Del Angel 1999). A few research groups have also found increased hydrogen pressure to be beneficial (Forni et al. 1997; Yuan and Keane 2003b). As discussed in Section 2.7, Forni et al. (1997) found increased hydrogen partial pressure to be beneficial due to decreased catalyst deactivation in the HDH of PCBs in a semi-continuous system. Yuan and Keane (2003b) also found increased hydrogen pressure to be beneficial, but attributed this effect to the minimization of mass transfer limitations. 
No statistically significant trend is discernible within the confidence of the $k_{2}$ estimates. However, though the reaction rate coefficients do not increase monotonically with decreased hydrogen pressure, the $k_{2}$ values for $30 \mathrm{psi}\left(3.49 \mathrm{hr}^{-1}\right)$ and $10 \mathrm{psi}\left(2.95 \mathrm{hr}^{-1}\right)$ were both higher than the $k_{2}$ value for $50 \mathrm{psi}\left(2.50 \mathrm{hr}^{-1}\right)$. This suggests a similar effect to that observed by Jujjuri and Keane (2010), where increasing hydrogen pressure above a certain threshold can inhibit catalytic HDH reactions. However, as previously mentioned, the observed completion times for the conversion of $\mathrm{TeCB}$ to cyclohexane fell within the same range (8-12 hours) for all three hydrogen pressures tested. Thus, while the estimated reaction rate coefficients may indicate a slight hydrogen pressure effect, the observed completion times suggest that the reactions are not strongly dependent on hydrogen pressure in the range of 10-50 psi.

\subsection{Application to REACH Technology}

The reaction rates observed in batch reactor experiments will likely differ from those observed in the semi-continuous REACH system. The REACH system utilizes a stationary catalyst column, while the batch reaction bottle is mechanically shaken during experiments. Additionally, due to the agitation of the reaction bottle, some metal particles detached from the surface of the catalyst and were suspended in solution during batch experiments. It is therefore possible that some mechanical deactivation occurred that might have decreased the observed reaction rates. Conversely, the surface area of the catalysts may have increased, thereby increasing the reaction rates. Nevertheless, the batch reactor data allow for a comparison of HDH and hydrogenation reaction rates with different solvent compositions and hydrogen pressures.

While a solvent composition of 67:33 was found to promote the fastest $\mathrm{HDH}$ and hydrogenation reaction rates, it is not necessarily the ideal solvent composition for the REACH technology due to the dual function of the solvent in both the extraction of contaminants from 
soil and in the liquid-phase catalytic treatment of contaminants. Wee and Cunningham (2013) found that 1,2,4,5-tetrachlorobenzene was not fully extracted from soil when the fraction of water in the solvent was greater than $50 \%$. In contrast, pentachlorophenol (PCP) was readily extracted with as high as $67 \%$ water by volume in the solvent. The findings presented in this thesis suggest that a higher fraction of water in the REACH solvent should be utilized when possible to maximize catalytic reaction rates. This conclusion is supported by the general consensus in the literature that adding water to a pure alcohol solvent improves the kinetics of heterogeneous catalytic HDH (Hoke et al. 1992; Benítez and Del Angel 1999; Xia et al. 2003; Concibido et al. 2006, 2007; Pozan and Boz 2008; Gómez-Quero et al. 2010a).

The TeCB data suggest that the rate of $\mathrm{HDH}$ is inversely related to hydrogen pressure in the range of 10-50 psi. While the significance of hydrogen pressure to hydrogenation reaction rates is unclear, complete conversion of $\mathrm{TeCB}$ to cyclohexane was achieved at hydrogen pressures as low as 5 psi. This finding suggests that the semi-continuous REACH system could be operated at lower hydrogen pressures than previously utilized (Wee and Cunningham 2011). Operation at lower hydrogen gas pressures would further improve the safety of the REACH technology. 


\section{CHAPTER 6: CONCLUSIONS AND FUTURE WORK}

\subsection{Summary and Conclusions}

The effects of the solvent composition and hydrogen pressure on the Pd-catalyzed hydrodehalogenation $(\mathrm{HDH})$ of 1,2,4,5-tetrachlorobenzene (TeCB) to benzene and the Rhcatalyzed hydrogenation of benzene to cyclohexane were investigated in a series of batch reactor experiments. An apparent first-order kinetic model, which followed the model development of Wee and Cunningham (2008), was developed for sequential HDH and hydrogenation reactions in a dual-catalyst batch reactor. Three water-to-ethanol solvent compositions $(33: 67,50: 50$, 67:33) were explored while holding hydrogen pressure constant at 50 psi. Three hydrogen pressures were investigated (50 psi, $30 \mathrm{psi}, 10 \mathrm{psi}$ ) while holding solvent composition constant at 50:50 water/ethanol. An additional experiment was conducted at 5 psi to test for a minimum hydrogen pressure required for the complete conversion of TeCB to cyclohexane.

Complete conversion of TeCB to cyclohexane was observed for all experimental conditions tested. The data were consistent with an apparent first order kinetic model for both Pd-catalyzed HDH of TeCB and Rh-catalyzed hydrogenation of benzene. Increased water-toethanol solvent composition ratios were hypothesized to increase catalytic reaction rates due to increased adsorption of hydrophobic reactants to the catalyst surface. As expected, HDH reaction rate coefficients increased monotonically with an increasing fraction of water in the solvent, though a greater increase was observed when the percentage of water was increased from $50 \%$ to $67 \%$ than when the percentage of water was increased from $33 \%$ to $50 \%$. The observed times for 
complete conversion of TeCB to cyclohexane correspondingly decreased from 12-18 hours with a $33 \%$ water solvent to $8-12$ hours with a $50 \%$ water solvent and to $1-1.5$ hours with a $67 \%$ water solvent. This effect was previously noted by Wee and Cunningham (2008) for the HDH of $\mathrm{TeCB}$, and is consistent with the general consensus in the literature that water addition to ethanol promotes faster HDH reaction rates (Benítez and Del Angel 1999; Concibido et al. 2006, 2007; Wee and Cunningham 2008; Gómez-Quero et al. 2010a).

Interestingly, similar hydrogenation reaction rate coefficient estimates were obtained from experiments conducted with $33 \%$ and $50 \%$ water solvent compositions. The water fraction in the solvent therefore did not appear to influence the hydrogenation reaction rate coefficients until the percentage of water in the solvent was increased from 50\% to $67 \%$. However, due to variability in the data, it cannot be said with $95 \%$ confidence that the reaction rate coefficient increased. The effect of solvent composition on the hydrogenation reaction rate coefficients observed in this study is similar to that observed by Concibido et al. (2006) for the HDH of tetrachloroethylene (PCE) in mixtures of water and ethanol. Concibido et al. (2006) found that PCE HDH reaction rates were independent of the proportion of water in a water/ethanol solvent until at least $50 \%$ water by volume was added.

It was hypothesized that catalytic reactions would be zero order with hydrogen pressure above a certain threshold, and that catalytic reactions would decrease with decreasing hydrogen pressure beneath the threshold. This hypothesis was based on the assumptions that hydrogen adsorption would follow the Langmuir adsorption isotherm, and that increased surface coverage of hydrogen on the catalyst would result in higher reaction rates. Interestingly, HDH reaction rate coefficients increased slightly but significantly with decreasing hydrogen pressure in the range of 10-50 psi. This can be attributed to the increased displacement of TeCB molecules from the 
catalyst surface with increased hydrogen pressure. This effect was noted previously by Jujjuri and Keane (2010) for the HDH of chlorinated benzenes.

In contrast to the $\mathrm{HDH}$ reaction rate coefficients, no statistically significant trend was observed within the $95 \%$ confidence intervals of the hydrogenation reaction rate coefficient estimates. Thus, the data suggest that hydrogenation reaction rates are independent of hydrogen pressure in the range of $10-50$ psi. The observed times for complete conversion of TeCB to cyclohexane were 8-12 hours at all three hydrogen pressures (50 psi, 30 psi, 10 psi), which suggests that the observed HDH hydrogen pressure effect was minor. An additional experiment was conducted at 5 psi to determine whether or not the reactions would reach completion at a lower hydrogen pressure. Complete conversion of TeCB to cyclohexane was achieved after 8 hours at 5 psi hydrogen pressure.

Some general conclusions can be made about the significance of water/ethanol solvent compositions and hydrogen pressures for the REACH technology. A higher percentage of water should be used in the water/ethanol solvent in the REACH system when possible to maximize catalytic reaction rates. The data also suggest that the REACH system can operate at pressures as

low as 5 psi, which would further improve the safety of the technology. The data further suggest that operation at lower hydrogen pressures may slightly improve HDH reaction rates.

\subsection{Future Work}

Catalytic conversion of HHOCs is a fundamental component of the REACH system. There are many opportunities for future research to expand upon the current understanding of the catalytic hydrodehalogenation and hydrogenation of HHOCs, and thus to further develop the REACH technology. Future work will focus on the semi-continuous REACH system, and could 
explore the effects of catalyst metal and support selection, co-pollutants, and solvent $\mathrm{pH}$ on catalytic reaction rates and catalyst deactivation.

Catalyst deactivation remains a significant challenge for environmental catalytic treatment technologies such as REACH. Ideally, catalysts would be reused indefinitely in the REACH process. However, catalyst deactivation has been observed with a Florida soil in the semi-continuous REACH system (Wee and Cunningham 2011). Thus, future work could explore the stability and longevity of the catalysts used in the REACH technology, especially if the catalyst must be routinely regenerated throughout its lifetime. The effects of operating parameters such as hydrogen pressure on catalyst deactivation behavior could also be investigated, since an optimal hydrogen pressure has not yet been established for the technology.

The effects of operating parameters on catalyst deactivation were also not explored in this thesis. Hoke et al. (1992) contend that an increased fraction of water in solvents improves the longevity of HDH catalysts. Alternatively, should a catalyst become irreversibly deactivated, the feasibility of recovering palladium and rhodium from spent catalysts could be explored as an alternative to disposal of the entire catalyst. Finally, solvent longevity could also be explored in future research. The effects of the accumulation of cyclohexane over time as the end-product in the catalytic conversion of TeCB in the REACH solvent have not yet been investigated. 


\section{REFERENCES CITED}

Akpa, B. S., D’Agostino, C., Gladden, L. F., Hindle, K., Manyar, H., McGregor, J., Li, R., Neurock, M., Sinha, N., Stitt, E. H., Weber, D., Zeitler, J. A., and Rooney, D. W. (2012). "Solvent effects in the hydrogenation of 2-butanone." J. Catal., 289, 30-41.

Aramendía, M. A., Boráu, V., García, C., Jiménez, F., Lafont, A., Marinas, J. M, and Urbano, F. J. (2002). "Liquid-phase hydrodechlorination of chlorobenzene over palladium-supported catalysts: influence of $\mathrm{HCl}$ formation and $\mathrm{NaOH}$ addition." J. Mol. Catal. A Chem., 184, 237-245.

Augustine, R. L. (1965). Catalytic Hydrogenation, Marcel Dekker, Inc., New York, NY.

Bae, J. W., Jang, E. J., Jo, D. H., Lee, J. S., and Lee, K. H. (2003). “Liquid-phase hydrodechlorination of $\mathrm{CCl}_{4}$ in a medium of ethanol with co-production of acetal and diethyl acetate." J. Mol. Catal. A Chem., 206, 225-238.

Bartholomew, C. H. (2001). "Mechanisms of catalyst deactivation.” Appl. Catal. A Gen., 212, $17-60$.

Benítez, J. L., and Del Angel, G. (1999). "Catalytic hydrodechlorination of chlorobenzene in liquide phase." React. Kinet. Catal. Lett., 66(1), 13-18.

Benítez, J. L., and Del Angel, G. (2000). "Effect of chlorine released during hydrodechlorination of chlorobenzene over Pd, Pt, and Rh catalysts.” React. Kinet. Catal. Lett., 70(1), 67-72.

Chaplin, B. P., Reinhard, M., Schneider, W. F., Scüth, C., Shapley, J. R., Strathmann, T. J., and Werth, C. J. (2012). "Critical review of Pd-based catalytic treatment of priority contaminants in water.” Environ. Sci. Technol., 46(7), 3655-3670.

Chen, I. M., Wanitchapichat, W., Jirakittayakorn, T., Sanohniti, S., Sudjarid, W., Wantawin, C., Voranisarakul, J., and Anotai, J. (2010). "Hexachlorobenzene dechlorination by indigenous sediment microorganisms." J. Hazard. Mater., 177, 244-250.

Chinchen, G. C. (1985). “Activation and deactivation of heterogeneous catalysts." Selected Developments in Catalysis, Jennings, J. R., ed., Blackwell Scientific Publications, Oxford, England. 
Concibido, N. C., Okudo, T., Nakano Y, Nishijimi W, and Okada M. (2005). "Enhancement of the catalytic hydrodechlorination of tetrachloroethylene in methanol at mild conditions by water addition." Tetrahedron Lett., 46, 3613-3617.

Concibido, N. C., Okuda, T., Nishijima W., and Okada, M. (2006). "Making the liquid-phase catalytic hydrodechlorination of PCE over Pd/C safer.” React. Kinet. Catal. Lett., 89(2), 369-376.

Concibido, N. C., Okuda, T., Nishijima W., and Okada, M. (2007). "Kinetics of deactivation of $\mathrm{Pd} / \mathrm{C}$ catalyst repeatedly used in the liquid-phase hydrodechlorination of PCE." React. Kinet. Catal. Lett., 90(1), 127-136.

Crittenden, J.C., Trussell, R. R., Hand, D. W., Howe, K. J., Tchobanoglous, G., and Borchardt, J. H. (2012). Water Treatment: Principles and Design, $3^{\text {rd }}$ ed., John Wiley \& Sons, Inc., Hoboken, NJ.

Duan, H., Wang, D., Koub, Y., and Li, Y. (2013). "Rhodium-nickel bimetallic nanocatalysts: high performance of room-temperature hydrogenation." Chem. Commun., 49, 303-305.

Fajt, V., Kurc, L., and Červený, L. (2008). "The effect of solvents on the rate of catalytic hydrogenation of 6-Ethyl-1,2,3,4-tetrahydroanthracene-9,10-dione.” Int. J. Chem. Kin., 40(5), 240-252.

Forni P, Prati L, and Rossi M. (1997). "Catalytic dehydrohalogenation of polychlorinated biphenyls - Part II: Studies on a continuous process.” Appl. Catal. B Environ., 14, 49-53.

Freifelder M. (1978). Catalytic Hydrogenation in Organic Synthesis: Procedures and Commentary, John Wiley \& Sons, Inc., New York, NY.

Gomes, H. I., Dias-Ferreira, C. D., and Ribeiro, A. B. (2012). "Electrokinetic remediation of organochlorines in soil: Enhancement techniques and integration with other remediation technologies." Chemosphere, 87, 1077-1090.

Gómez-Quero, S., Díaz, E., Cárdenas-Lizana, F., and Keane, M. A. (2010a). "Solvent effects in the catalytic hydrotreatment of haloaromatics over $\mathrm{Pd} / \mathrm{Al}_{2} \mathrm{O}_{3}$ in water+organic mixtures." Chem. Eng. Sci., 65, 3786-3797.

Gómez-Quero, S., Cárdenas-Lizana, F., and Keane, M. A. (2010b). "Solvent effects in the hydrodechlorination of 2,4,-dichlorophenol over $\mathrm{Pd} / \mathrm{Al}_{2} \mathrm{O}_{3}$." AIChE J., 56(3), 756-767.

Harada, T., Ikeda, S., Ng, Y. H., Sakata, T., Mori, H., Torimoto, T., and Matsumura, M. (2008). "Rhodium nanoparticle encapsulated in a porous carbon shell as an active heterogeneous catalyst for aromatic hydrogenation." Adv. Funct. Mater., 18, 2190-2196. 
Hashimoto, Y., Uemichi, Y., and Ayame, A. (2005). "Low-temperature hydrodechlorination of chlorobenzenes on palladium-supported alumina catalysts." J. Jpn. Pet. Inst., 48(3), 127-136.

Hegedus, L. L., and McCabe, R. W. (1984). Catalyst Poisoning, Marcel Dekker, Inc., New York, NY.

Hoke, J. B., Gramiccioni, G. A., and Balko, E. N. (1992). "Catalytic hydrodechlorination of chlorophenols." Appl. Catal. B Environ., 1(4), 285-296.

Ishihara, S., Ido, A., Monguchi, Y., Nagase, H., and Sajiki, H. (2012). "Pd/C-catalyzed dechlorination of polychlorinated biphenyls under hydrogen gas-free conditions." $J$. Hazard. Mater., 229-230, 15-19.

Jacinto, M. J., Kiyohara, P. K., Masunaga, S. H., Jardim, R. F., and Rossi, L. M. (2008). "Recoverable rhodium nanoparticles: Synthesis, characterization and catalytic performance in hydrogenation reactions." Appl. Catal. A Gen., 338, 52-57.

Janiak, T., and Okal, J. (2009). "Effectiveness and stability of commercial Pd/C catalysts in the hydrodechlorination of meta-substituted chlorobenzenes." Appl. Catal. B Environ., 92, 384-392.

Jujjuri, S., and Keane, M. A. (2010). "Catalytic hydrodechlorination at low hydrogen partial pressures: Activity and selectivity response.” Chem. Eng. J., 157, 121-130.

Khodadoust, A. P., Suidan, M. T., Acheson, C. M., and Brenner, R. C. (1999). "Solvent extraction of pentachlorophenol from contaminated soils using water-ethanol mixtures." Chemosphere, 38: 2681-2693.

Konuspayev, S. R., Schaimardan, M., and Murzin, D. Y. (2009). "Kinetics of liquid-phase benzene hydrogenation on Rh/C.” Res. Chem. Intermed., 35, 1-11.

Kopinke, F., Mackenzie, K., Koehler, R., and Georgi, A. (2004). "Alternative sources of hydrogen for hydrodechlorination of chlorinated organic compounds in water on Pd catalysts." Appl. Catal. A Gen., 271, 119-128.

Kopinke, F., Angeles-Wedler, D., Fritsch, D., and Mackenzie, K. (2010). "Pd-catalyzed hydrodechlorination of chlorinated aromatics in contaminated waters-Effects of surfactants, organic matter and catalyst protection by silicone coating." Appl. Catal. B Environ., 96, 323-328.

Kovenklioglu, S., Cao, Z., Shah, D., Farrauto, R. J., and Balko, E. N. (1992). "Direct catalytic hydrodechlorination of toxic organics in wastewater." AIChE J., 38(7), 1003-1012. 
Kume, A., Monguchi, Y., Hattori, K., Nagase, H., and Sajiki, H. (2008). "Pd/C-catalyzed practical degradation of PCBs at room temperature." Appl. Catal. B Environ., 81(3-4), 274-282.

Lassová, L., Lee, H. K., and Hor, T. S. (1999). "Catalytic dechlorination of chlorobenzenes: effect of solvent on efficiency and selectivity." J. Mol. Catal. A Chem., 144, 397-403.

Lee, H. H. (1985). "Physical Deactivation and Sintering." Heterogeneous Reactor Design, E. B. Nauman, ed., Butterworth Publishers, Boston, MA, 201-233.

Lee, M., Cord-Ruwisch, R., and Manefield, M. (2010). "A process for the purification of organochlorine contaminated activated carbon: Sequential solvent purging and reductive dechlorination." Water Res., 44, 1580-1590.

Léger, B., Nowicki, A., Roucoux, A., and Rolland, J. (2007). "Competitive hydrogenation/ dehalogenation of halogenoarenes with surfactant-stabilized aqueous suspensions of rhodium and palladium colloids: A major effect of the metal nature." J. Mol. Catal. A Chem., 266(1/2), 221-225.

Li, Y. S., and LeBoeuf, E. J. (2010). "Investigation of transport- and sorption-related factors on the removal of hydrophobic organic compounds in heterogeneous soils using a hierarchical modeling approach.” Transp. Porous Med., 84, 319-333.

Lowry, G. V., and Reinhard, M. (2000). "Pd-catalyzed TCE dechlorination in groundwater: solute effects, biological control, and oxidative catalyst regeneration." Environ. Sci. Technol., 34(15), 3217-3223.

Mackenzie, K. Frenzel, H., and Kopinke, F. D. (2006). "Hydrodehalogenation of halogenated hydrocarbons in water with Pd catalysts: Reaction rates and surface competition." Appl. Catal. B Chem., 63, 161-167.

Maegawa, T., Akashi, A., Yaguchi, K., Iwasaki, Y., Shigetsura, M., Monguchi, Y., and Sajiki, H. (2009). "Efficient and practical arene hydrogenation by heterogeneous catalysts under mild conditions." Chem. Eur. J. 15: 6953-6963.

Moulijn, J. A., Van Diepen, A. E., and Kapteijn, F. (2001). "Catalyst deactivation: is it predictable? What to do?" Appl. Catal. A Gen., 212, 3-16.

Munakata, N., Roberts, P. V., Reinhard, M., and McNab, W. W. (1998). "Catalytic dechlorination of halogenated hydrocarbon compounds using supported palladium: a preliminary assessment of matrix effects." Proceedings of the GQ'98 Groundwater Quality: Remediation and Protection Conference, IAHS, Tübingen, Germany.

Murena, F., and Gioia, F. (2009). "Solvent extraction of chlorinated compounds from soils and hydrodechlorination of the extract phase." J. Hazard. Mater., 162, 661-667. 
Navon, R., Eldad, S., Mackenzie, K., and Kopinke, F. (2012). "Protection of palladium catalysts for hydrodechlorination of chlorinated organic compounds in wastewaters." Appl. Catal. B Environ., 119-120, 241-247.

Nazaroff, W. W., and Alvarez-Cohen, L. (2001). Environmental Engineering Science, John Wiley \& Sons, Inc., New York, NY.

Ordóñez, S., Vivas, B. P., and Díez, F. V. (2010). "Minimization of the deactivation of palladium catalysts in the hydrodechlorination of trichloroethylene in wastewaters." Appl. Catal. B Environ., 95, 288-296.

Osborn, C. J. (2011). "Catalytic hydrodehalogenation and hydrogenation of halogenated aromatic organic contaminants for application to soil remediation." M.S. thesis, Univ. of South Florida, Tampa, FL.

Potrawfke, T., Timmis, K. N., and Wittich, R. M. (1998). "Degradation of 1,2,3,4tetrachlorobenzene by pseudomonas chloraraphis RW71." Appl. Env. Microbiol., 64(10), 3798 .

Pozan, G. S., and Boz, I. (2008). "Hydrodechlorination of 2,3,5-Trichlorophenol in Methanol/Water on Carbon Supported Pd-Rh Catalysts.” Environ. Eng. Sci., 25(8), 1197-1202.

Rivera-Austrui, J., Borrajo, M. A., Martinez, K., Adrados, M. A., Abalos, M., Bavel, B. V., Rivera, J., and Abad, E. (2011). "Assessment of polychlorinated dibenzo-p-dioxin and dibenzofuran emissions from a hazardous waste incineration plant using long-term sampling equipment." Chemosphere, 87(9), 1343-1349.

Satterfield, C. N. (1991). Heterogeneous Catalysis in Industrial Practice, 2nd ed., McGraw-Hill, Inc., New York, NY.

Scüth, C., and Reinhard, M. (1998). "Hydrodechlorination and hydrogenation of aromatic compounds over palladium-on-alumina in hydrogen-saturated water." Appl. Catal. B Environ., 18, 215-221.

Shindler, Y., Matatov-Meytal, Y., and Sheintuch, M. (2001). "Wet hydrodechlorination of $p$ chlorophenol using Pd supported on an activated carbon cloth." Ind. Eng. Chem. Res., 40, 3301-3308.

Sidhpuria, K. B., Parikh, P. A., Bahadur, P., Tyagi, B., and Jasra, R. V. (2009). "Influence of the surface acidity of ZSM-5 support on the catalytic activity of Rh/ZSM-5 for hydrodearomatization of toluene." Catal. Today, 141, 12-18.

Singh, U. K., and Vannice, M. A. (2001). "Kinetics of liquid-phase hydrogenation reactions over supported metal catalysts - a review." Appl. Catal. A Gen., 213, 1-24 
Söderström, G., and Marklund, S. (2002). "PBCDD and PBCDF from incineration of wastecontaining brominated flame retardants." Environ. Sci. Technol., 36(9), 1959-1964

Smith, G. V., and Notheisz, F. (1999). Heterogeneous Catalysis in Organic Chemistry, Academic Press, San Diego, CA.

Stanislaus, A., and Cooper, B. H. (1994). "Aromatic hydrogenation catalysis: a review." Catal. Rev., 36(1), 75-123.

Tang, W. Z. (2004). Physicochemical Treatment of Hazardous Wastes, CRC Press LLC, Boca Raton, FL.

Ticknor, J. L. (2012). “Analysis and remediation of chlorinated hydrocarbons in environmental media." M.S. thesis, Univ. of South Florida, Tampa, FL.

Urbano, F. J., and Marinas, J. M. (2001). "Hydrogenolysis of organohalogen compounds over palladium supported catalysts." J. Mol. Catal. A Chem., 173, 329-345.

U.S. EPA. (2001). “A Citizen's Guide to Solvent Extraction.” EPA-542-F-01-009, <http://www.epa.gov> (May 6, 2013).

U.S. EPA. (2011). "Common chemicals found at Superfund sites." Superfund, <http://www.epa.gov/oerrpage/superfund/health/contaminants/radiation/chemicals.htm> (May 14, 2013).

U.S. EPA. (2012a). "Priority chemicals." Hazardous Waste - Waste Minimization, $<$ http://www.epa.gov/osw/hazard/wastemin/priority.htm> (May 6, 2013).

U.S. EPA. (2012b). "Priority pollutants." Water: CWA Methods, $<$ http://water.epa.gov/scitech/methods/cwa/pollutants.cfm> (May 6, 2013).

U.S. National Library of Medicine (NLM). (2003). “1,2,4,5-Tetrachlorobenzene.” Hazardous Substances Data Bank, <http://toxnet.nlm.nih.gov/cgi-bin/sis/htmlgen?HSDB> (May 20, 2013).

Wee, H. Y. (2007). "Remedial extraction and catalytic hydrodehalogenation for treatment of soils contaminated by halogenated hydrophobic organic compounds." Doctoral Dissertation, Texas A\&M University, College Station, TX.

Wee, H. Y., and Cunningham, J. A. (2008). "Palladium-catalyzed hydrodehalogenation of 1,2,4,5-tetrachlorobenzene in water-ethanol mixtures." J. Hazard. Mater., 155, 1-9.

Wee, H. Y., and Cunningham, J. A. (2011). "Remediation of contaminated soil by solvent extraction and catalytic hydrodehalogenation: Semicontinuous process with solvent recycle.” Environ. Prog. Sustainable Energy, 30, 589-598. 
Wee, H. Y., and Cunningham, J. A. (2013). "Soil treatment by solvent extraction and catalytic hydrodehalogenation." Int. J. Environ. Waste Manage., 11, 59-74.

Wu, B., Chen, H., Wang, S. J., Wai, C. M., Liao, W., and Chiu K. (2012). "Reductive dechlorination for remediation of polychlorinated biphenyls." Chemosphere, 88(7), 757768.

Xia, C., Xu, J., Wu, W., Luo, Q., Chen, J., Zhang, Q., and Liang, X. (2003). “Catalytic hydrodechlorination of 2,4,4'-trichloro-2'-hydroxydiphenylether under mild conditions." Appl. Catal. B Environ., 45, 281-292.

Xia, C., Xu, J., Wu, W., and Liang, X. (2004). "Pd/C-catalyzed hydrodehalogenation of aromatic halides in aqueous solutions at room temperature under normal pressure." Catal. Commun., 5, 383-386.

Xia, C., Liu, Y., Zhou, S., Yang, C., Liu, S., Xu, J., Yu, J., Chen, J., and Liang, X. (2009). “The $\mathrm{Pd}$-catalyzed hydrodechlorination of chlorophenols in aqueous solutions under mild conditions: a promising approach to practical use in wastewater." J. Hazard. Mater., 169, 1029-1033.

Yakovlev, V. A., Terskikh, V. V., Simagina, V. I., and Likholobov, V. A. (2000). "Liquid phase catalytic hydrodechlorination of chlorobenzene over supported nickel and palladium catalysts: an NMR insight into solvent function.” J. Molec. Catal. A Chem., 153, 231236.

Yuan, G., and Keane, M. A. (2003a). "Catalyst deactivation during the liquid phase hydrodechlorination of 2,4-dichlorophenol over supported Pd: influence of the support." Catal. Today, 88, 27-36.

Yuan, G., and Keane, M. A. (2003b). "Liquid phase catalytic hydrodechlorination of 2,4dichlorophenol over carbon supported palladium: an evaluation of transport limitations." Chem. Eng. Sci., 58, 257-267. 
APPENDICES 


\section{Appendix A: Electron Capture Detector Gas Chromatographic Methods}

Table A-1. TeCB Gas Chromatography Analysis Method 1 (Before Replacement of ECD Anode and Column)

\begin{tabular}{|c|c|}
\hline Detector & Electron Capture Detector (ECD) \\
\hline Column & J\&W DB-WAX \\
\hline Run Time & 15 min. \\
\hline Syringe Washes & $\begin{array}{l}1 \text { pre-injection solvent wash (pentane) } \\
3 \text { pre-injection sample washes } \\
2 \text { post-injection solvent washes (pentane) }\end{array}$ \\
\hline Injection & $\begin{array}{l}0.5 \mathrm{~L} \text { liquid by autosampler at normal speed } \\
4 \text { sample pumps }\end{array}$ \\
\hline Sampling Rate & $25 \mathrm{pts} / \mathrm{s}$ \\
\hline Oven Temperature Program & $\begin{array}{l}\text { Initial temperature }=60^{\circ} \mathrm{C} \text { for } 1 \mathrm{~min} \text {. } \\
\text { Increase } 5^{\circ} \mathrm{C} / \mathrm{min} \text {. to } 100^{\circ} \mathrm{C} \text {. Hold for } 0 \mathrm{~min} \text {. } \\
\text { Increase } 10^{\circ} \mathrm{C} / \mathrm{min} \text {. to } 115^{\circ} \mathrm{C} \text {. Hold for } 4.5 \mathrm{~min} \text {. }\end{array}$ \\
\hline ECD Temperature & $230^{\circ} \mathrm{C}$ \\
\hline Injection Port Temperature & $230^{\circ} \mathrm{C}$ \\
\hline Helium Flow Rate & $3.00 \mathrm{~mL} / \mathrm{min}$. \\
\hline Split Ratio & $35: 1$ \\
\hline Range (1 or 20 ) & 1 \\
\hline Attenuation (0 to -6$)$ & -2 \\
\hline
\end{tabular}

Table A-2. TeCB Gas Chromatography Analysis Method 2 (After Replacement of ECD Anode and Column)

\begin{tabular}{|l|l|}
\hline Detector & Electron Capture Detector (ECD) \\
\hline Column & $\mathrm{J} \& \mathrm{~W}$ HP-5 \\
\hline Run Time & 15 min. \\
\hline Syringe Washes & $\begin{array}{l}1 \text { pre-injection solvent wash (pentane) } \\
3 \text { pre-injection sample washes } \\
2 \text { post-injection solvent washes (pentane) }\end{array}$ \\
\hline Injection & $\begin{array}{l}0.5 \mathrm{~L} \text { liquid by autosampler at normal speed } \\
4 \text { sample pumps }\end{array}$ \\
\hline Sampling Rate & 25 pts/s \\
\hline Oven Temperature Program & $\begin{array}{l}\text { Initial temperature }=60^{\circ} \mathrm{C} \text { for } 1 \text { min. } \\
\text { Increase } 5^{\circ} \mathrm{C} / \text { min. to } 100^{\circ} \mathrm{C} \text {. Hold for } 0 \text { min. } \\
\text { Increase } 10^{\circ} \mathrm{C} / \text { min. to } 115^{\circ} \mathrm{C} \text {. Hold for } 4.5 \text { min. }\end{array}$ \\
\hline ECD Temperature & $230^{\circ} \mathrm{C}$ \\
\hline Injection Port Temperature & $230^{\circ} \mathrm{C}$ \\
\hline Helium Flow Rate & $3.00 \mathrm{~mL} / \mathrm{min}$. \\
\hline Split Ratio & $20: 1$ \\
\hline Range (1 or 20) & 1 \\
\hline Attenuation (0 to -6$)$ & -6 \\
\hline
\end{tabular}




\section{Appendix B: Flame Ionization Detector Gas Chromatographic Methods}

Table B-1. Cyclohexane and Benzene Gas Chromatography Analysis Method 1

\begin{tabular}{|l|l|}
\hline Detector & Flame Ionization Detector (FID) \\
\hline Column & RTX-1301 \\
\hline Run Time & $14 \mathrm{~min}$. \\
\hline Injection & $1 \mathrm{~mL}$ headspace by manual injection \\
\hline Sampling Rate & $12.5 \mathrm{pts} / \mathrm{s}$ \\
\hline Oven Temperature & $\begin{array}{l}\text { Initial temperature }=30^{\circ} \mathrm{C} \text { for } 8 \mathrm{~min} . \\
\text { Increase } 20^{\circ} \mathrm{C} / \text { min. to } 130^{\circ} \mathrm{C} \text {. Hold for } 1 \mathrm{~min} .\end{array}$ \\
\hline FID Temperature & $230^{\circ} \mathrm{C}$ \\
\hline Injection Port Temperature & $230^{\circ} \mathrm{C}$ \\
\hline Helium Flow Rate & $3.00 \mathrm{~mL} / \mathrm{min}$. \\
\hline Split Ratio & OFF until timed event (SPL1) \\
\hline Range (1 or 20) & 1 \\
\hline Attenuation $(\mathbf{0}$ to $-\mathbf{6})$ & 0 until timed event (ATT1) \\
\hline Offset & $5.0 \mathrm{mV}$ \\
\hline Timed Events & $\begin{array}{l}\text { SPL1 }=50 \text { at } 0.5 \mathrm{~min} . \\
\text { ATT1 }=-5 \text { at } 12 \text { min. }\end{array}$ \\
\hline
\end{tabular}

Table B-2. Cyclohexane and Benzene Gas Chromatography Analysis Method 2

\begin{tabular}{|l|l|}
\hline Detector & Flame Ionization Detector (FID) \\
\hline Column & RTX-1301 \\
\hline Run Time & $15.19 \mathrm{~min}$. \\
\hline Injection & $1 \mathrm{~mL}$ headspace by manual injection \\
\hline Sampling Rate & $12.5 \mathrm{pts} / \mathrm{s}$ \\
\hline Oven Temperature & $\begin{array}{l}\text { Initial temperature }=30^{\circ} \mathrm{C} \text { for } 6 \mathrm{~min} . \\
\text { Increase } 13^{\circ} \mathrm{C} / \mathrm{min} . \text { to } 130^{\circ} \mathrm{C} \text {. Hold for } 1.5 \mathrm{~min} .\end{array}$ \\
\hline FID Temperature & $230^{\circ} \mathrm{C}$ \\
\hline Injection Port Temperature & $230^{\circ} \mathrm{C}$ \\
\hline Helium Flow Rate & $3.00 \mathrm{~mL} / \mathrm{min}$. \\
\hline Split Ratio & OFF until timed event (SPL1) \\
\hline Range (1 or 20) & 1 \\
\hline Attenuation $(\mathbf{0}$ to $-\mathbf{6})$ & 0 until timed event (ATT1) \\
\hline Offset & $5.0 \mathrm{mV}$ \\
\hline Timed Events & $\begin{array}{l}\text { SPL1 }=40 \text { at } 0.5 \mathrm{~min} . \\
\text { ATT1 }=-5 \text { at } 12 \text { min. }\end{array}$ \\
\hline
\end{tabular}

